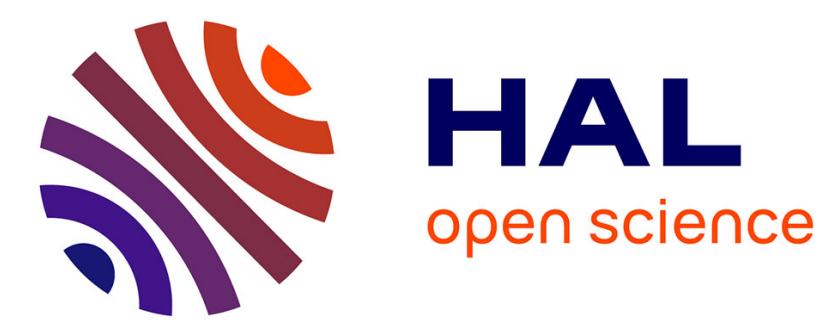

\title{
Perfectly Matched Absorbing Layers for the Paraxial Equations
}

\author{
Francis Collino
}

\section{To cite this version:}

Francis Collino. Perfectly Matched Absorbing Layers for the Paraxial Equations. [Research Report] RR-2964, INRIA. 1996. inria-00073734

\section{HAL Id: inria-00073734 https://hal.inria.fr/inria-00073734}

Submitted on 24 May 2006

HAL is a multi-disciplinary open access archive for the deposit and dissemination of scientific research documents, whether they are published or not. The documents may come from teaching and research institutions in France or abroad, or from public or private research centers.
L'archive ouverte pluridisciplinaire HAL, est destinée au dépôt et à la diffusion de documents scientifiques de niveau recherche, publiés ou non, émanant des établissements d'enseignement et de recherche français ou étrangers, des laboratoires publics ou privés. 
INSTITUT NATIONAL DE RECHERCHE EN INFORMATIQUE ET EN AUTOMATIQUE

\title{
Perfectly Matched Absorbing Layers for the Paraxial Equations
}

\author{
F. Collino
}

$\mathbf{N}^{\circ} 2964$

Août 1996

THÈME 4 



\title{
Perfectly Matched Absorbing Layers for the Paraxial Equations
}

\author{
F. Collino \\ Thème 4 - Simulation et optimisation \\ de systèmes complexes \\ Projet Ondes
}

Rapport de recherche no2964 — Août 1996 - 40 pages

\begin{abstract}
A new absorbing boundary technique for the paraxial wave equations is proposed and analyzed. Numerical results show the efficiency of the method.
\end{abstract}

Key-words: Paraxial equation, Numerical reflexion coefficient, Migration, Schroedinger equation, Perfectly matched layer, absorbing layers

(Résumé : tsvp)

This work has been performed as part of the IFP-INRIA $\psi$ Consortium Project. This paper has been submited to the Journal of Computational Physics 


\section{Couches absorbantes parfaitement adaptées pour les équations paraxiales}

Résumé : Une nouvelle technique de conditions absorbantes pour les équations paraxiales est présentée et analysée. L'idée est d'interpréter puis de généraliser le modèle de couches proposé par J.P. Bérenger pour l'électromagnétisme aux équations de type Schroedinger. Quelques résultats numériques montrent la potentialité de la méthode

Mots-clé : Equation paraxiale, coefficient de réflexion numérique, migration, equation de Schoedinger, Couches parfaitement adaptées, Couches absorbantes 
Eh oui les hirondelles

Et encore des billevesées

\section{Introduction}

Paraxial equations are now a classical tool in seismic processing. They are partial differential equations providing approximate solution to the one-way wave equation. Each equation is labelled by an angle that describes the cone of propagation directions that are correctly modelled. Claerbout, [5], was the first to introduce fifteen degree and forty-five degree type equations for the extrapolation of 2-D seismic data. Since then, many authors have developed the concept and the use of paraxial equations has been extended to various situations like heterogeneous or 3D media. However, the treatment of the artificial lateral boundaries, that is of great practical importance, has not given rise to a satisfactory answer up to now. The reason is probably that usual absorbing boundary conditions are not very well suited to this type of equations: as will be shown below, the decomposition of the propagation operator into out-going and in-going parts makes appear the non local operator $\sqrt{\partial_{z}}$ which is difficult to handle numerically. In this paper, we propose to adapt a novel technique, introduced for electromagnetism recently [4]. This technique consists in designing an absorbing layer called perfectly matched layer (PML) which possesses the astonishing property of generating no reflection at the interface between the free medium and the artificial absorbing medium. This property allows us to use a very high damping parameter inside the layer and consequently a small layer length while achieving a quasi-perfect absorption of the waves.

\section{The fifteen degree paraxial equation}

We start with the migration of a single seismic trace (point source) by the simplest paraxial equation, say, the 15 degree approximation. The formulation of the problem is

$$
\text { find } \quad m(x, z)=\frac{1}{\pi} \Re e\left(\int_{0}^{\infty} \hat{S}(\omega) v e^{i \omega\left(t_{s}-z / c\right)} d \omega\right), \quad(c \equiv 1)
$$

RR no2964 
where $v$ satisfies the 15 degree paraxial equation

$$
2 i \omega \partial_{z} v(x, z)-\partial_{x x} v(x, z)=\delta\left(x-x_{s}\right) \delta(z) .
$$

$\hat{S}(\omega)$ is the Fourier transform of the source trace, $x_{s}$ its location, $t_{s}$ the time of explosion and the velocity is set to one. (2) is a Schrödinger like equation. Its solution is (cf. appendix A)

$$
v(x, z)=\sqrt{\frac{-i}{8 \pi \omega z}} e^{-i \omega \frac{\left(x-x_{s}\right)^{2}}{2 z}}, \quad \sqrt{-i}=e^{-i \frac{\pi}{4}} .
$$

In [2], a transparent condition is derived; it is shown that the following Dirichlet to Neumann relation holds

$$
\left(\partial_{x} v+\sqrt{2 i \omega} \sqrt{\partial_{z}} v\right)_{/ x=0}=0,
$$

where $\sqrt{\partial_{z}}$ is the half derivative operator

$$
\sqrt{\partial_{z}} \varphi=\frac{1}{\pi} \partial_{z} \int_{0}^{z} \frac{\varphi(s)}{\sqrt{z-s}} d s .
$$

Condition (4) is first order with respect to the variable $z$ and thus can be employed as a boundary condition for practical computation. The main drawback is due to the non local character of the operator $\sqrt{\partial_{z}}$ : a convolution in $z$ at the artificial boundary must be performed, [12]. An alternative method, proposed by Di Menza, [8], consists in substituting in (4) some approximation of $\sqrt{\partial_{z}}$ by rational fractions,

$$
\sqrt{\partial_{z}} \approx \sum_{\ell=1}^{L} a_{\ell}^{L} \frac{\partial_{z}}{\partial_{z}+d_{\ell}^{L}},
$$

where $a_{\ell}, d_{\ell}, \ell=1, . ., L$ are real numbers characterizing the approximation and $L$ some integer large enough to obtain a good approximation. The absorbing boundary condition thus obtained can be written as a system of partial differential equations involving auxiliary functions (one per fraction),

$$
\left\{\begin{array}{l}
\left(\partial_{x} v\right)_{/ x=0}+\sqrt{2 i \omega}\left(\sum_{\ell=1}^{L} a_{\ell}^{L} v_{/ x=0}-\sum_{\ell=1}^{L} a_{\ell}^{L} d_{\ell}^{L} \varphi_{\ell}\right)=0, \\
\partial_{z} \varphi_{\ell}+d_{\ell}^{L} \varphi_{\ell}=v_{/ x=0}, \forall \ell=1, . ., L
\end{array}\right.
$$


Numerical experiments show that a large $L$ is required to achieve good absorption of the waves at the boundary. Comparing with the wave equation, where first or second order conditions often give good results, the case of the Schrödinger equation appears to be more difficult. The reason is that the symbol of the operator $\sqrt{\partial_{z}}$, say $\sqrt{i k_{z}}$, does not fit very well with rational or polynomial functions at infinity, $k_{z} \rightarrow \infty$ (compare with $i \omega \sqrt{1-k_{x}^{2} / \omega^{2}}, \omega \rightarrow \infty$ for the wave equation).

We present now an alternative to absorbing boundary conditions based on the construction of an appropriate absorbing boundary layer model. The idea comes from a reinterpretation of Bérenger's PML as a change of variable in the complex plane (see appendix B and [14]). For designing our PMLs model, we consider a positive function $\sigma(s),(\sigma(s)$ will play the role of a damping factor) and assume

$$
\sigma(x)=\left\{\begin{array}{ll}
0 & \text { for } \quad x<0 \\
\geq 0 & \text { for } \quad x>0
\end{array} .\right.
$$

We define the change of variables,

$$
\tilde{x}=x-\frac{i}{\omega} \int_{0}^{x} \sigma(s) d s,
$$

and consider the function

$$
\tilde{v}(x, z)=v(\tilde{x}, z)=\sqrt{\frac{-i}{8 \pi \omega z}} \exp \left(-i \omega \frac{\left(\tilde{x}-x_{s}\right)^{2}}{2 z}\right) .
$$

Note that condition $\sigma(s) \equiv 0$ for $s<0$ implies

$$
\tilde{v}(x, z)=v(x, z), \quad \forall x<0 .
$$

$\tilde{v}$ only differs from $v$ inside the absorbing layer $x>0$ where it is really damped as

$$
|\tilde{v}(x, z)|=\sqrt{\frac{1}{8 \pi \omega z}} \exp \left(-\frac{\left(x-x_{s}\right)}{z} \int_{0}^{x} \sigma(s) d s\right) \longrightarrow 0, \quad \text { as } x \rightarrow \infty .
$$

Now, we look for a partial differential equation satisfied by $\widetilde{v}$. From

$$
\frac{\partial^{2} v}{\partial x^{2}}(x=\tilde{x})=\left(\frac{\partial \tilde{x}}{\partial x}\right)^{-1} \frac{\partial}{\partial x}\left(\left(\frac{\partial \tilde{x}}{\partial x}\right)^{-1} \frac{\partial \tilde{v}}{\partial x}\right)(x)
$$

RR no2964 
we get

$$
2 i \omega \partial_{z} \tilde{v}-\frac{i \omega}{i \omega+\sigma} \partial_{x}\left(\frac{i \omega}{i \omega+\sigma} \partial_{x} \tilde{v}\right)=\delta\left(x-x_{s}\right) \delta(z) .
$$

Equation (14) is the sought layer model for the Schrödinger equation (2). It consists in the simple modification

$$
\frac{\partial}{\partial x} \longrightarrow \frac{\partial}{\partial \tilde{x}}=\frac{i \omega}{i \omega+\sigma(x)} \frac{\partial}{\partial x}
$$

in the initial equation. This change does not alter the solution in the area of interest $(v=\tilde{v}$ for $x<0)$. This important property is confirmed by a plane wave analysis; the plane wave solutions of

$$
2 i \omega \partial_{z} \tilde{v}-\frac{i \omega}{i \omega+\sigma} \partial_{x}\left(\frac{i \omega}{i \omega+\sigma} \partial_{x} \tilde{v}\right)=0
$$

are sought in the form

$$
\begin{cases}\tilde{v}(x, z)=\left(e^{-i k_{x} x}+R e^{i k_{x} x}\right) e^{i k_{z} z} & x<0 \\ \tilde{v}(x, z)=T e^{-i k_{x} \tilde{x}} e^{i k_{z} z} & x>0\end{cases}
$$

where $k_{x}$ is assumed to be positive (right-going plane wave). $T$ is the transmission coefficient, $R$ the reflection coefficient. (16) is satisfied if the dispersion relation

$$
k_{x}^{2}=-2 \omega k_{z}, \quad k_{x}>0,
$$

is fulfilled. Coefficients $T$ and $R$ are determined by the interface conditions: as the quantity on which the operator $\partial_{x}$ is applied in equation (16) must be continuous (cf. appendix C), we have

$$
\begin{cases}\tilde{v}\left(0^{+}, z\right) & =\tilde{v}\left(0^{-}, z\right) \\ \left(1-i \frac{\sigma\left(0^{+}\right)}{\omega}\right)^{-1} \partial_{x} \tilde{v}\left(0^{+}, z\right) & =\left(1-i \frac{\sigma\left(0^{-}\right)}{\omega}\right)^{-1} \partial_{x} \tilde{v}\left(0^{-}, z\right)\end{cases}
$$

and thus

$$
\left\{\begin{array} { l } 
{ T = 1 + R } \\
{ ( 1 - i \frac { \sigma ( 0 ^ { + } ) } { \omega } ) ^ { - 1 } ( - i k _ { x } ) \frac { \partial \tilde { x } } { \partial x } ( 0 ^ { + } ) T = - i k _ { x } ( 1 - R ) }
\end{array} \Rightarrow \left\{\begin{array} { l } 
{ T = 1 + R } \\
{ T = 1 - R }
\end{array} \Rightarrow \left\{\begin{array}{l}
T=1 \\
R=0
\end{array} .\right.\right.\right.
$$


The reflection coefficient is zero and the transmission is total for all right-going plane waves. In this sense the layer is perfectly matched.

For practical considerations, we cannot work with the absorbing half-space $x>0$, but we must deal with a finite length absorbing layer. If $\delta$ is the length of the layer, the system of equations is closed with a Dirichlet boundary condition

$$
\left\{\begin{array}{l}
2 i \omega \partial_{z} \tilde{v}(x, z)-\frac{i \omega}{i \omega+\sigma} \partial_{x}\left(\frac{i \omega}{i \omega+\sigma} \partial_{x} \tilde{v}(x, z)\right)=0, \quad x<\delta \\
\tilde{v}(x=\delta, z)=0
\end{array}\right.
$$

The same plane wave analysis now gives

$$
\begin{cases}\tilde{v}(x, z)=\left(e^{-i k_{x} x}+R e^{i k_{x} x}\right) e^{i k_{z} z} & x<0 \\ \tilde{v}(x, z)=(1+R) \frac{e^{-i k_{x}(\tilde{x}-\tilde{\delta})}-e^{i k_{x}(\tilde{x}-\tilde{\delta})}}{e^{i k_{x} \tilde{\delta}}-e^{-i k_{x} \tilde{\delta}}} e^{i k_{z} z} & 0<x<\delta,\end{cases}
$$

where $k_{x}, k_{z}$ satisfy (18). As $\tilde{v}$ as given by (22) is continuous, $R$ is determined by the second transmission condition

$$
-i k_{x}(1+R) \frac{e^{i k_{x} \tilde{\delta}}+e^{-i k_{x} \tilde{\delta}}}{e^{i k_{x} \tilde{\delta}}-e^{-i k_{x} \tilde{\delta}}}=-i k_{x}(1-R),
$$

or

$$
R=-e^{-2 i k_{x} \tilde{x}(\delta)}, \quad \Rightarrow|R|=e^{-2 \frac{k_{x}}{\omega} \int_{0}^{\delta} \sigma(s) d s} .
$$

(24) is the same expression as the one obtained by Bérenger for the electromagnetic problem, [4]. In this case, $R$ is the reflection coefficient of the "layer" in the sense that the reflection coefficient at $x=0$ is zero, the reflection coefficient at $x=\delta$ is -1 and $R$ describes the percentage of the original wave amplitude after two passes through the PML. The specificity of the wave propagation model only appears in the expression for $k_{x}$, as given by the dispersion relation.

Let us come back to the point source problem (i.e. problem (21) with $\delta\left(x-x_{s}\right)$ on the right hand side, $\left.x_{s}<0\right)$. The solution can be evaluated by the image principle of geometrical optics and is given by

$$
\tilde{v}(x, z)=\sqrt{\frac{-i}{8 \pi \omega z}} \exp \left(-i \omega \frac{\left(\tilde{x}-x_{s}\right)^{2}}{2 z}\right)-\tilde{v}_{r}(x, z) .
$$

RR no2964 
$\tilde{v}_{r}$ is the mirror image of $v$ with respect to the plane $\{x=\delta\}$. It is the reflected wave due to the Dirichlet Boundary condition and is given by

$$
\left\{\begin{array}{l}
\tilde{v}_{r}(x, z)=\sqrt{\frac{-i}{8 \pi \omega z}} \exp \left(-i \omega \frac{\left(\breve{x}-\left(2 \delta-x_{s}\right)\right)^{2}}{2 z}\right) \\
\breve{x}=x-\frac{i}{\omega} \int_{2 \delta}^{x} \breve{\sigma}(s) d s, \quad \breve{\sigma}(s)= \begin{cases}\sigma(s), & s<\delta \\
\sigma(2 \delta-s), & s>\delta\end{cases}
\end{array}\right.
$$

or

$\tilde{v}_{r}(x, z)=\sqrt{\frac{-i}{8 \pi \omega z}} \exp \left(-i \frac{\omega}{2 z}\left(x+x_{s}-2 \delta+i \int_{0}^{\delta} \frac{\sigma(s)}{\omega} d s+i \int_{x}^{\delta} \frac{\sigma(s)}{\omega} d s\right)^{2}\right)$

and, in particular if $x \leq 0$

$$
\left|\tilde{v}_{r}\right|=\sqrt{\frac{1}{8 \pi \omega z}} \exp \left(-4 \frac{\delta}{z} \int_{0}^{\delta} \sigma(s) d s\right) \exp \left(2 \frac{\left(x+x_{s}\right)}{z} \int_{0}^{\delta} \sigma(s) d s\right) .
$$

This implies

if $z \neq 0, \quad(v-\tilde{v})\left(x, z ; x_{s}\right)=\tilde{v}_{r} \rightarrow 0$ uniformly in $x, x_{s} \leq 0$ as $\int_{0}^{\delta} \sigma(s) d s \rightarrow \infty$.

In other terms, the reflection due to the finite length of the layer can be made arbitrarily small by choosing $\sigma(s)$ large enough.

From a mathematical point of view, the PML model can be seen as an evolution variational problem

$$
\left\{\begin{array}{l}
\text { Find } u: z \in] 0, Z[\longrightarrow u(., z) \in V \quad \text { such that } \forall v(x) \in V \\
2 i \omega \frac{d}{d z}\left(\int u(x, z) v(x) \frac{d x}{d(x)}\right)=-\int \partial_{x} u(x, z) \partial_{x} v(x) d(x) d x
\end{array}\right.
$$

where $V$ is the set of functions $v(x)$ such that both $|v|^{2}$ and $\left|\partial_{x} v\right|^{2}$ are integrable with $v(\delta)=0$ (it can be shown that functions of $V$ are continuous). Problem (30) is initialized at $z=0$ by an initial condition

$$
u(z=0)=u^{0} \in V
$$


An approximation of problem (30), (31) can be constructed by considering a sequence of finite dimensional subspaces of $V$, say $V_{h}$, with

$$
\overline{\bigcup_{h>0} V_{h}}=V, \quad, V_{h^{\prime}} \subset V_{h}, \text { if } h^{\prime}<h,
$$

and replacing $V$ by $V_{h}, u^{0}$ by its projection $u_{0}^{h}$ onto $V_{h}$. It is not difficult to prove that the solution, $u_{h}(z)$, exists and is the solution of ordinary differential equations with constant coefficients. Unfortunately, no estimation is easily available except when $d(x)$ is independent of $x$ (see Appendix D). The technical difficulty is the existence of the imaginary part of $d$ making the usual technique apparently useless. So, passing to the limit as $h \rightarrow 0$ is still an open problem and further investigations are necessary to establish existence and uniqueness.

\section{Extension to higher order paraxial equations}

The higher-order paraxial equations are constructed from approximations of the square root by rational functions, [1]

$$
\sqrt{1-\tau}=1-\sum_{\ell=1}^{L} \beta_{\ell} \frac{\tau}{1-\alpha_{\ell} \tau}
$$

$L$ is the order of the approximation. The case $L=1, \beta_{1}=\frac{1}{2}, \alpha_{1}=0$ corresponds to the previously studied fifteen degree approximation. When (32) is used to evaluate the operator $\sqrt{1+\omega^{-2} \partial_{x x}}$, equation (2) must be replaced by

$$
\begin{cases}\partial_{z} v=i \omega \sum \beta_{\ell} \varphi_{\ell} & x \in R, z>0 \\ \omega^{2} \varphi_{\ell}+\partial_{x x} \psi_{\ell}=0, \quad \psi_{\ell}=\alpha_{\ell} \varphi_{\ell}+v, & x \in R, z>0 .\end{cases}
$$

In order to ensure uniqueness, an appropriate radiation condition at infinity or a limiting absorption (i.e., replace $\omega$ by $\omega+i \epsilon$ and pass to the limit $\epsilon \rightarrow 0^{-}$, cf. [10]), must be used. In addition, an initial condition at $z=0$, say $v(x, z=$ $0)=v_{0}(x)$ is supposed to be given. The construction of the PML model is 
very easy to perform: we change the system (33) into

$$
\left\{\begin{array}{lc}
\partial_{z} \tilde{v}=i \omega \sum \beta_{\ell} \tilde{\varphi}_{\ell} & x \leq \delta, z>0 \\
\omega^{2} \tilde{\varphi}_{\ell}+\frac{i \omega}{\sigma(x)+i \omega} \partial_{x}\left(\frac{i \omega}{\sigma(x)+i \omega} \partial_{x} \tilde{\psi}_{\ell}\right)=0 & x \leq \delta, z>0 \\
\tilde{\psi}_{\ell}=\alpha_{\ell} \tilde{\varphi}_{\ell}+\tilde{v} & x \leq \delta, z>0 \\
\tilde{\psi}_{\ell}(\delta, z)=0 & z>0,
\end{array}\right.
$$

that is, we use formally the substitution

$$
\frac{\partial}{\partial x} \longrightarrow \frac{\partial}{\partial \tilde{x}}=\frac{i \omega}{i \omega+\sigma(x)} \frac{\partial}{\partial x}
$$

As the closed form for the Green function is not known, in contrast to the 15 degree approximation, cf (3), we cannot proceed as above to prove that $\tilde{v} \rightarrow v$ for $x \leq 0$ as $\int \sigma(s) d s$ increases toward infinity. However, the plane wave analysis describing what happens at a boundary still holds. Indeed, all the required arguments for proving that the reflection coefficient vanishes do not depend on the particular structure of the equation governing the wave: the form of the dispersion relation do not play any role. Let us make this assertion precise:

the particular plane wave solutions are

$$
\tilde{\varphi}_{\ell}=\frac{k_{x}^{2}}{\omega^{2}-\alpha_{\ell} k_{x}^{2}} \cdot \tilde{v}
$$

where $\tilde{v}$ is given by $(22)$ and $k_{x}$ is a solution of

$$
k_{z}=-\sum_{\ell=1}^{L} \beta_{\ell} \frac{k_{x}^{2}}{\omega^{2}-\alpha_{\ell} k_{x}^{2}} .
$$

The condition of transmission (i.e. the continuity of $\frac{i \omega}{i \omega+\sigma} \partial_{x} \psi_{\ell}$ at $x=0$ ) provides the reflection coefficient

$$
R=-e^{2 i k_{x} \tilde{x}(\delta)}, \quad \Rightarrow|R|=e^{-2 \frac{k_{x}}{\omega} \int_{0}^{\delta} \sigma(s) d s}
$$


which is nothing else than (24).

A direct inspection of equation (38) would lead us to pick the largest possible $\sigma$ that would allow us to obtain a reflection as weak as desired. However, this nice property is no longer true when the discretization scheme for numerically solving the equations is used. As a matter of fact, and as we will see below, the discretization of the equations induces a shift between the points located near the interface and thus the layer is only approximately matched. The numerical dispersion induces a reflection at the interface which is roughly proportional to $\sigma \Delta x$. We are facing the situation known from classical layer models, cf. [13], where a balance has to be found between choosing $\sigma$ not too high (this would create a reflection at the free-medium/absorbing-medium interface) and picking $\sigma$ not too low (this would yield an insufficient damping of the waves returned by the Dirichlet conditions).

\section{Discretization of the perfectly matched layer}

We begin with a very simple 1D model and consider a right side PMLs model for the equation

$$
\omega^{2} \lambda u+\partial_{x x} u=f, \quad x \in R
$$

namely

$$
\left\{\begin{array}{l}
\omega^{2} \lambda u+\frac{i \omega}{i \omega+\sigma} \partial_{x}\left(\frac{i \omega}{i \omega+\sigma} \partial_{x} u\right)=f, \quad x \leq \delta \\
u(x=\delta)=0,
\end{array}\right.
$$

where $\lambda$ is a parameter, $\sigma(s) \equiv 0$ for $s<0$ and $f(s) \equiv 0$ for $s \geq 0$.

Let $\Delta x$ be a discretization step, $\gamma$ a positive parameter less than $\frac{1}{4}$ (the parameter $\gamma$ is introduced to improve the approximation of the second derivative operator). The discretized scheme is

$$
\left\{\begin{array}{c}
\omega^{2} \lambda\left(\frac{1-2 \gamma}{2}\left(\frac{1}{d_{j-\frac{1}{2}}}+\frac{1}{d_{j+\frac{1}{2}}}\right) u_{j}+\frac{\gamma}{d_{j+\frac{1}{2}}} u_{j+1}+\frac{\gamma}{d_{j-\frac{1}{2}}} u_{j-1}\right) \\
+\frac{1}{\Delta x}\left(d_{j+\frac{1}{2}} \frac{u_{j+1}-u_{j}}{\Delta x}-d_{j-\frac{1}{2}} \frac{u_{j}-u_{j-1}}{\Delta x}\right)=f_{j},
\end{array}\right.
$$

where

$$
d_{j+\frac{1}{2}}=\frac{i \omega}{i \omega+\sigma_{j+\frac{1}{2}}}, \quad \sigma_{j+\frac{1}{2}}=\frac{1}{\Delta x} \int_{j \Delta x}^{(j+1) \Delta x} \sigma(s) d s
$$

RR no2964 
This scheme can be viewed as the discretization of the variational formulation

$$
\begin{aligned}
& \lambda \omega^{2} \int \frac{1}{d(x)} u(x) \varphi(x) d x-\int d(x) \partial_{x} u(x) \partial_{x} \varphi(x) d x=\int f(x) \varphi(x) d x, \\
& \forall \varphi(x) \in \mathrm{H}^{1}(]-\infty, \delta[), \varphi(\delta)=0, \quad d(x)=\frac{i \omega}{i \omega+\sigma},
\end{aligned}
$$

by piecewise linear finite elements, the mass matrix being a linear combination between the exact matrix and the lumped mass matrix (cf. Appendix E).

The scheme is analyzed by a classical discrete plane wave analysis: particular solutions of scheme (41) with zero right hand side are sought in the form

$$
u_{j}=e^{-i k_{x} j \Delta x}+R e^{i k_{x} j \Delta x}, \quad j \leq 0 .
$$

For $j<0$ the scheme reduces to

$$
\omega^{2} \lambda \Delta x^{2}\left((1-2 \gamma) u_{j}+\gamma\left(u_{j+1}+u_{j-1}\right)\right)+\left(u_{j+1}-2 u_{j}+u_{j-1}\right)=0 .
$$

Substituting (44) into (45), $k_{x}$ is found to satisfy the numerical dispersion relation

$$
\frac{4}{\Delta x^{2}} \sin ^{2}\left(\frac{k_{x} \Delta x}{2}\right)=\lambda \omega^{2}\left(1-4 \gamma \sin ^{2}\left(\frac{k_{x} \Delta x}{2}\right)\right) .
$$

We distinguish between two situations. First, we assume $\sigma$ to be constant and $\delta$ rejected to infinity, later we will investigate the case of a finite length layer. In the first case, the solution satisfies

$$
\omega^{2} \lambda \Delta x^{2}\left((1-2 \gamma) u_{j}+\gamma\left(u_{j+1}+u_{j-1}\right)\right)+d_{\sigma}^{2}\left(u_{j+1}-2 u_{j}+u_{j-1}\right)=0, \quad \forall j>0,
$$

with

$$
d_{\sigma}=\frac{i \omega \Delta x}{\sigma \Delta x+i \omega \Delta x}
$$

and the solution can be found in the form

$$
u_{j}=(1+R) e^{-i k_{x}^{\sigma} j \Delta x}, \quad j \geq 0,
$$

with

$$
\frac{4}{\Delta x^{2}} \sin ^{2}\left(\frac{k_{x}^{\sigma} \Delta x}{2}\right)=\lambda \omega^{2}\left(1-4 \gamma \sin ^{2}\left(\frac{k_{x}^{\sigma} \Delta x}{2}\right)\right)\left(1+\frac{\sigma \Delta x}{i \omega \Delta x}\right)^{2} .
$$


It is easy to verify that each equation of the scheme is satisfied at nodes $j \neq 0$ (i.e. in the homogeneous parts) as soon as the two dispersion relations (46) and (50) are fulfilled. Then, the remaining equation at $j=0$,

$$
\left\{\begin{array}{l}
\omega^{2} \lambda\left(\frac{1-2 \gamma}{2}\left(\frac{1}{d_{\sigma}}+1\right) u_{0}+\gamma u_{1}+\frac{\gamma}{d_{\sigma}} u_{-1}\right)+\frac{1}{\Delta x}\left(\frac{u_{1}-u_{0}}{\Delta x}-d_{\sigma} \frac{u_{0}-u_{-1}}{\Delta x}\right)=0 \\
\text { with } u_{0}=1+R, u_{-1}=e^{i k_{x} \Delta x}+R e^{-i k_{x} \Delta x}, u_{1}=(1+R) e^{-i k_{x}^{\sigma} \Delta x}
\end{array}\right.
$$

provides the reflection coefficient $R$ :

$$
\begin{aligned}
& R=-\frac{c_{0}+\tilde{d}_{\frac{1}{2}} e^{-i k_{x}^{\sigma} \Delta x}+\tilde{d}_{-\frac{1}{2}} e^{i k_{x} \Delta x}}{c_{0}+\tilde{d}_{\frac{1}{2}} e^{-i k_{x}^{\sigma} \Delta x}+\tilde{d}_{-\frac{1}{2}} e^{-i k_{x} \Delta x}} \\
& c_{0}=\omega^{2} \lambda \Delta x^{2} \frac{1-2 \gamma}{2}\left(\frac{1}{d_{\sigma}}+1\right)-d_{\sigma}-1 \\
& \tilde{d}_{\frac{1}{2}}=d_{\sigma}+\omega^{2} \lambda \Delta x^{2} \frac{\gamma}{d_{\sigma}}, \quad \tilde{d}_{-\frac{1}{2}}=1+\omega^{2} \lambda \Delta x^{2} \gamma,
\end{aligned}
$$

Thanks to MAPLE, we get

$$
R=-\frac{\lambda}{16}(\sigma \Delta x-2 i \omega \Delta x) \sigma \Delta x(1+2 \sqrt{\lambda} \gamma \sigma \Delta x)+O\left(\Delta x^{4}\right),
$$

which confirms that the discretization induces a dispersive numerical reflection the numerical layer is only approximately matched and $\sigma$ must not be picked too high.

In the case of a layer with a finite length and composed of $n_{\ell}$ layers characterized by

$$
\tilde{\sigma}=\left(\sigma_{\frac{1}{2}}, \sigma_{\frac{3}{2}}, \ldots, \sigma_{n_{\ell}-\frac{1}{2}}\right),
$$

the equations

$$
\left\{\begin{array}{c}
\omega^{2} \lambda\left(\frac{1-2 \gamma}{2}\left(\frac{1}{d_{j+\frac{1}{2}}}+\frac{1}{d_{j-\frac{1}{2}}}\right) u_{j}+\frac{\gamma}{d_{j+\frac{1}{2}}} u_{j+1}+\frac{\gamma}{d_{j-\frac{1}{2}}} u_{j-1}\right) \\
+\frac{1}{\Delta x}\left(d_{j+\frac{1}{2}} \frac{u_{j+1}-u_{j}}{\Delta x}-d_{j-\frac{1}{2}} \frac{u_{j}-u_{j-1}}{\Delta x}\right)=0, \\
d_{j+\frac{1}{2}}=\frac{i \omega \Delta x}{i \omega \Delta x+\sigma_{j+\frac{1}{2}} \Delta x}
\end{array}\right.
$$

RR no2964 
are now verified for $j \leq n_{\ell}-1$ with the additional condition $u_{n_{\ell}}=0$. This system of $n_{\ell}$ equations can be viewed as a tridiagonal linear system. The unknowns are $u_{0}, u_{1}, \ldots, u_{n_{\ell}-1}$ and the second term is a vector all of whose components are zero except for the first one which is proportional to $u_{-1}$.

Let us define

$$
M=\left[\begin{array}{cccccc}
c_{1} & \tilde{d}_{\frac{3}{2}} & & & & \\
\tilde{d}_{\frac{1}{2}} & c_{2} & \tilde{d}_{\frac{3}{2}} & & & \\
& \tilde{d}_{\frac{3}{2}} & \cdots & \ldots & & \\
& & \cdots & & & \\
& & & & \ldots & \tilde{d}_{n_{\ell}-\frac{1}{2}} \\
& & & & \tilde{d}_{n_{\ell-\frac{1}{2}}} & c_{n_{\ell}-1}
\end{array}\right], \quad F=\left[\begin{array}{c}
1+\gamma \lambda \omega^{2} \Delta x^{2} \\
0 \\
\cdot \\
\cdot \\
\cdot \\
0
\end{array}\right.
$$

with

$$
\left\{\begin{array}{l}
c_{j}=\omega^{2} \lambda \Delta x^{2} \frac{1-2 \gamma}{2}\left(\frac{1}{d_{j+\frac{1}{2}}}+\frac{1}{d_{j-\frac{1}{2}}}\right)-d_{j+\frac{1}{2}}-d_{j-\frac{1}{2}} \\
\tilde{d}_{j+\frac{1}{2}}=d_{j+\frac{1}{2}}+\omega^{2} \lambda \Delta x^{2} \frac{\gamma}{d_{j+\frac{1}{2}}}
\end{array}\right.
$$

The equations inside the layer are solved according to

$$
\hat{U}={ }^{t}\left[u_{0}, u_{1}, \ldots ., u_{n_{\ell}-1}\right]=-u_{-1} M^{-1} \cdot F
$$

(note that $d_{j+\frac{1}{2}} \equiv 1$ when $j<0$ ).

Now if we look for a solution in the form

$$
u_{j}=e^{-i k_{x} j \Delta x}+R e^{i k_{x} j \Delta x}, \quad j \leq 0
$$

where $k_{x}$ satisfies (46), we immediately obtain from (58)

$$
(1+R)=u_{0}=-{ }^{t} F \cdot M^{-1} \cdot F u_{-1}=-{ }^{t} F \cdot M^{-1} \cdot F\left(e^{-i k_{x} \Delta x}+R e^{i k_{x} \Delta x}\right),
$$

or

$$
R=R(\lambda, \omega \Delta x ; \tilde{\sigma} \Delta x)=\frac{1-\left({ }^{t} F \cdot M^{-1} \cdot F\right) e^{-i k_{x} \Delta x}}{1-\left({ }^{t} F \cdot M^{-1} \cdot F\right) e^{i k_{x} \Delta x}} .
$$

Formula (61) gives the numerical reflexion coefficient for the finite length layer. 
To illustrate the influence of the discretization on the reflexion coefficient, the following example is considered,

$$
\begin{cases}\lambda=1, & \text { (1D Helmoltz equation) } \\ \delta=2 \pi / \omega & \text { (layer length }=1 \text { wavelength) } \\ \sigma(x)=\sigma_{c}(x / \delta)^{2}, \text { for } x>0 & \text { (parabolic law) } \\ \sigma_{c}=\frac{3}{2 \delta} \log \left(\frac{1}{R_{0}}\right) & R_{0}=10^{-2}, 10^{-3}, 10^{-4}\end{cases}
$$

( $\sigma_{c}$ is picked such that the reflexion coefficient for the continuous model as given by $(24)$ with $k_{x}=\omega$ is equal to $R_{0}$ ). In figure 1 , one sees the variation of the numerical reflexion coefficient (61) versus the number of points per wavelength $N=2 \pi /(\omega \Delta x)$ (which is equal, in this case, to the number of points in the layer as the length layer is one wavelength). First, it can be verified that the numerical reflexion coefficient converges to $R_{0}$, the value derived for the continuous model, when the number of points per wavelength increases: our scheme is found to be consistent. Second, the convergence of the scheme appears to be slower for the small values of $R_{0}$ than for the largest one: the theoretical reflexion coefficient $R_{0}$ is approximately reached for $N \approx 5$ when $R_{0}=0.01, N \approx 10$ when $R_{0}=0.001$ and $N \approx 35$ when $R_{0}=0.0001$. Of course, the larger the number of points in the layer is, the smaller the reflexion coefficient can be. Finally, we remark that, when $N$ is fixed, the smallest reflexion coefficient is not necessarily obtained for the largest value of $\sigma_{c}$. This emphasizes the important role played by the numerical dispersion.

Let us return to the higher-order paraxial equations described in (33). The discretization of the problem is based upon the following principles, cf. [9], [6]:

- Use splitting techniques to reduce the $L$ fractions problem to a serie of $L$ problems with a unique fraction.

- Integrate in $z$ with an implicit and second order accurate Crank-Nicolson scheme for the $z$ direction (classical explicit schemes in $z$ are unstable). We consider $z$ as an evolution variable.

- Perform the discretization in $x$ through a variational approach using a uniform mesh and a finite elements/finite differences method.

RR no2964 
For simplicity, we restrict the problem to the case $L=1, \alpha_{1} \equiv \alpha, \beta_{1} \equiv \beta$. The integration scheme is

$$
\left\{\begin{array}{l}
\frac{v^{k+1}-v^{k}}{\Delta z}=i \omega \beta \varphi^{k+\frac{1}{2}} \\
\omega^{2} \mathbf{M} \varphi^{k+\frac{1}{2}}-\mathbf{K} \psi^{k+\frac{1}{2}}=0 \\
\psi^{k+\frac{1}{2}}=\alpha \varphi^{k+\frac{1}{2}}+\frac{v^{k+1}+v^{k}}{2} .
\end{array}\right.
$$

In these expressions, $v^{k}=\left(v_{j}^{k}\right)$ denotes the approximate value of the unknown $\tilde{v}$ at depth $k \Delta z$ and lateral position $j \Delta x\left(v^{k}\right.$ is supposed to be piecewise linear in $x) \cdot \varphi^{k+\frac{1}{2}}=\left(\varphi_{j}^{k+\frac{1}{2}}\right)$ and $\psi^{k+\frac{1}{2}}=\left(\psi_{j}^{k+\frac{1}{2}}\right)$ are defined similarly. $\mathbf{M}$ and $\mathbf{K}$ are the matrices arising from the variational equalities

$$
\omega^{2} \int \frac{1}{d(x)} \varphi^{k+\frac{1}{2}} \hat{v} d x-\int d(x) \psi^{k+\frac{1}{2}} \hat{v} d x=0, \quad \forall \hat{v} \in P^{1}
$$

[9], [6] with the assembly described in appendix E. It is possible to eliminate the unknown $\varphi^{k+\frac{1}{2}}$ and to get

$$
\left\{\begin{array}{l}
\frac{v^{k+1}-v^{k}}{\Delta z}=i \omega \frac{\beta}{\alpha} \psi^{k+\frac{1}{2}}-i \omega \frac{\beta}{\alpha} \frac{v^{k+1}+v^{k}}{2} \\
\omega^{2} \mathbf{M} \psi^{k+\frac{1}{2}}-\alpha \mathbf{K} \psi^{k+\frac{1}{2}}=\omega^{2} \mathbf{M} \frac{v^{k+1}+v^{k}}{2} .
\end{array}\right.
$$

The discrete harmonic solutions of (65) are sought in the form

$$
\left\{\begin{array}{l}
\mathrm{v}^{k}=\mathbf{v} e^{-i k_{z} k \Delta z} \\
\psi^{k+\frac{1}{2}}=\Psi e^{-i k_{z}\left(k+\frac{1}{2}\right) \Delta z} .
\end{array}\right.
$$

Substituting (66) into (65) yields

$$
\left\{\begin{array}{l}
\frac{-2}{\Delta z} \sin \left(\frac{k_{z} \Delta z}{2}\right) \mathbf{v}=\omega \frac{\beta}{\alpha} \Psi-\omega \frac{\beta}{\alpha} \cos \left(\frac{k_{z} \Delta z}{2}\right) \mathbf{v} \\
\omega^{2} \mathbf{M} \Psi-\alpha \mathbf{K} \Psi=\omega^{2} \cos \left(\frac{k_{z} \Delta z}{2}\right) \mathbf{M} \mathbf{v}
\end{array}\right.
$$


or

$$
\mathbf{v}=\omega \beta\left(-\alpha \frac{2}{\Delta z} \sin \left(\frac{k_{z} \Delta z}{2}\right)+\beta \omega \cos \left(\frac{k_{z} \Delta z}{2}\right)\right)^{-1} \Psi
$$

and

$$
\omega^{2} \lambda \mathbf{M} \Psi-\mathbf{K} \Psi=0
$$

with

$$
\lambda\left(k_{z}\right)=\frac{-\frac{2}{\Delta z} \tan \left(\frac{k_{z} \Delta z}{2}\right)}{\beta \omega-\alpha \frac{2}{\Delta z} \tan \left(\frac{k_{z} \Delta z}{2}\right)} .
$$

In this expression, $k_{z}$ may be interpreted as the wave number in the $z$ direction seen from a system of coordinates moving downward in the positive $z$ direction with velocity 1 . In other words

$$
k_{z}=k_{z}^{\text {true }}-\omega=\omega(\cos (\theta)-1),
$$

where $\theta$ is the angle between the propagation direction and the vertical $z$ direction. Then

$$
\lambda=\frac{-k_{z}}{\omega \beta-\alpha k_{z}}+O\left(\Delta z^{2}\right) \approx \frac{1-\cos (\theta)}{\alpha+\beta-\alpha \cos (\theta)} .
$$

Equation (69) is exactly the numerical scheme we have studied previously (see (40)). The reflection induced by the boundary is characterized by the reflection coefficient $R(\lambda, \omega \Delta x ; \tilde{\sigma} \Delta x)$ given by (61). It is natural to choose $\tilde{\sigma}$ in order to minimize $R$ for all the angles of incidence contained in the incoming waves, say

$$
\tilde{\sigma}^{*}=\operatorname{Arg} \min \int_{0}^{\lambda^{+}}|R(\lambda, \omega \Delta x ; \tilde{\sigma} \Delta x)|^{2} d \lambda, \quad \lambda^{+}=\frac{1-\cos \left(\theta^{+}\right)}{\alpha \cos \left(\theta^{+}\right)-\alpha-\beta},
$$

or further, in order to get a discretized criterium,

$$
\left\{\begin{array}{l}
\tilde{\sigma}^{*}(N)=\operatorname{Arg} \min J(N ; \sigma \Delta x) \\
J(N ; \sigma \Delta x)=\frac{1}{P} \sum_{i=1}^{P}\left|R\left(\lambda_{i}, \omega \Delta x=\frac{1}{2 \pi N} ; \sigma \Delta x\right)\right|^{2}, \quad \lambda_{i}=\frac{1-\cos \left(\frac{i}{P} \theta^{+}\right)}{\alpha \cos \left(\frac{i}{P} \theta^{+}\right)-\alpha-\beta} .
\end{array}\right.
$$

RR no2964 
We have used the famous software M2QN1, [11], to find the minima associated to the values

$$
\left\{\begin{array}{l}
\alpha=\frac{1}{4}, \beta=\frac{1}{2},(45 \text { degree approximation) } \\
\theta^{+}=90 \text { degree, } \Rightarrow \lambda^{+}=\frac{4}{3} \\
P=100 \\
\gamma=0.1 \text { (Claerbout's choice). }
\end{array}\right.
$$

The remaining parameter $N=(2 \pi \omega \Delta x)^{-1}$ has been taken equal to 5,7 , $10,15,20,30,40$. As a result, the optimal values, shown in Table 1 , depend weakly on $N$, the number of points per wavelength, and the mean value of the reflection coefficient is in any case less than $2 \%$. Furthermore, we note that this coefficient increases with the number of points per wavelength.

\begin{tabular}{c||c|c|c|c|c|c|c|} 
& $N=5$ & $N=7$ & $N=10$ & $N=15$ & $N=20$ & $N=30$ & $N=40$ \\
\hline$\sigma \Delta x(1)$ & 0.185 & 0.167 & 0.267 & 0.360 & 0.383 & 0.395 & 0.405 \\
\hline$\sigma \Delta x(2)$ & 0.652 & 0.521 & 0.474 & 0.376 & 0.440 & 0.5058 & 0.540 \\
\hline$\sigma \Delta x(3)$ & 1.539 & 1.273 & 1.265 & 0.981 & 0.793 & 0.853 & 0.890 \\
\hline$\sigma \Delta x(4)$ & 3.424 & 2.953 & 2.715 & 2.664 & 2.508 & 2.207 & 2.095 \\
\hline$\sigma \Delta x(5)$ & 9.909 & 9.249 & 8.886 & 8.257 & 7.995 & 7.885 & 7.830 \\
\hline$\frac{1}{100} \sum_{i=1}^{100}|R|_{i}$ & .0043 & .0050 & .0063 & .0085 & .0105 & 0.0126 & 0.0132 \\
\hline
\end{tabular}

Table 1: Values of the 5 optimal damping coefficients $\sigma \Delta x$ versus the number of points per wavelengths $N=(2 \pi \omega \Delta x)^{-1}$ and mean value of the reflexion coefficient modulus. These values correspond to the 45 degree paraxial equation with $\gamma=0.1$ and $\theta^{+}=90$ degree.

Figure 2 shows the values of the modulus of the reflection coefficient

$$
\left|R\left(\lambda(\theta), \frac{1}{2 \pi N} ; \tilde{\sigma} \Delta x(N)\right)\right|,
$$

versus the angle of propagation $\theta$ for different number of points per wavelength.

Note that, as there is no damping for the propagation at incidence along $z$, it is only natural that we find a higher coefficient for the low values of the angle. 
In Figure 3 are shown the values of the modulus of the reflection coefficient

$$
\left|R\left(\lambda(\theta), \frac{1}{2 \pi N} ; \tilde{\sigma} \Delta x(7)\right)\right|
$$

as a function of angle of propagation $\theta$ for various numbers of points per wavelength and a fixed value of $\sigma \Delta x$. The 5 corresponding values for $\sigma \Delta x$ are determined through the optimization process with 7 points per wavelength. In this case, although the values for $\sigma \Delta x$ are not optimal when the number of points per wavelengths is not 7 , the reflection coefficient remains in the vicinity of some few per cents for the angles larger then 10 degrees (note that a wave associated with $\theta$ equal to 10 degree corresponds to an incidence with respect to the normal of the absorbing layer of 80 degree).

These results are somewhat paradoxical: the paraxial equation is designed for waves propagating close to the $z$ direction, but to such waves correspond a large reflexion coefficient (see equation (24)). The problem is that the waves propagating vertically are grazing waves when viewed from the artificial boundary. We will return to this point in the numerical experiments.

\section{$5 \quad$ Numerical experiments}

In order to test the method, we have performed the migration of a point source. With a 45 degree paraxial equation, the problem can be written as follows

$$
\left\{\begin{array}{l}
\text { Find } m(x, z) \text { such that } \\
m(x, z)=\frac{1}{\pi} \int_{0}^{\infty} \Re e\left(v(x, z, \omega) e^{-i \omega z}\right) d \omega
\end{array}\right.
$$

RR no2964 
where

$$
\left\{\begin{array}{lc}
\partial_{z} v=i \frac{\omega}{2 c} \varphi & |x| \leq L, z>0 \\
\omega^{2} \frac{\sigma c+i \omega}{i \omega c} \varphi+\partial_{x}\left(\frac{i \omega c}{\sigma c+i \omega} \partial_{x} \psi\right)=0 & |x| \leq L, z>0 \\
\psi=\frac{1}{4} \varphi+v, & |x| \leq L z>0 \\
\psi( \pm L, z)=0 & z>0 \\
v(x, z=0)=v_{0} & |x|<L .
\end{array}\right.
$$

The velocity is $1000 \mathrm{~m} / \mathrm{s}$ and $v_{0}$, the initial condition at $z=0$, is a gaussian point source located in $x_{s}$ and given as

$$
\left\{\begin{array}{l}
v_{0}(x)=e^{-\left(\left(x-x_{s}\right) / 64\right)^{2}} \cdot \tilde{S}(\omega) \\
\tilde{S}(\omega)=i \frac{\omega}{\omega_{s}} e^{-\frac{\omega^{2}}{\omega_{s}^{2}}} e^{i \omega t_{s}}
\end{array}\right.
$$

The space steps $\Delta z$ and $\Delta x$ have been chosen equal to 12.5 meters such that one has a discretization of 11 points per wavelength for the frequency corresponding to the highest value of $\tilde{S}(\omega)$. We have considered 150 points in the $x$ direction and 100 points in the $z$ direction. The time of the explosion occurrence, $t_{s}$, is 82 per cent of $50 \Delta z / c$ and the frequency of the signal, $2 \pi \omega_{s}$, is $10 / t_{s}$. Finally, the integration over the frequencies has been performed by adding 120 regularly spaced samples up to $\frac{8}{3}\left(2 \pi \omega_{s}\right)$.

In Figure 4, we have represented four different migrated sections for two locations of the source and two boundary conditions. The first boundary condition corresponds to a Dirichlet boundary condition on both sides of the computational domain. The second one is computed using the PML model with 5 layers of length $\Delta x$ for the left side of the model. The 5 corresponding values for $\sigma \Delta x$ are determined through the optimization process described previously with 10 points per wavelength. The idea is to take the best coefficients for the frequency corresponding approximatively to the maximum of $\tilde{S}(\omega)$ (note that in this experiment $\sigma$ has been chosen independently of the frequency). 
We note that the strong reflection due to the Dirichlet condition cannot be seen any more with the absorbing layer. Even when the source is located rather close to the boundary ( 15 nodes in $x$ ), the PML layer allows us to obtain a pretty good result (see also the third column of Table 2 for quantitative measures). Furthermore, as only 4 extra nodes have been necessary to construct the numerical absorbing layer, the cost induced by the PML is really marginal.

In Figure 5, similar experiments have been performed with the 60 degree paraxial equation. The same 5 damping coefficients as for the 45 degree paraxial equation have been used. As a result, the use of the PML allows us to eliminate the reflected wave, although the coefficients were not designed for the 60 degree paraxial equation.

More generally, we have performed several experiments with values of $\sigma(i) \Delta x, i=1, . .5$ other than the optimal ones and we have found that the method is not very sensitive to the precise value of the coefficients. It is mainly the order of magnitude of the coefficients that is important.

To quantify the results, a reference solution $\tilde{m}$ has been computed in the large domain $]-L+2 x_{s}, L[\times] 0, Z[$ (the left boundary has been moved from 0 to $-L+2 x_{s}$ where no reflexion can occur) and compared with $m$. The following error norms are considered

$$
\begin{aligned}
& \epsilon_{2}=\left(\sum_{i} \sum_{k}\left(m_{i}^{k}-\tilde{m}_{i}^{k}\right)^{2}\right)^{1 / 2} \cdot\left(\sum_{i} \sum_{k}\left(\tilde{m}_{i}^{k}\right)^{2}\right)^{-1 / 2} \\
& \epsilon_{\infty}=\sup _{i, k}\left|m_{i}^{k}-\tilde{m}_{i}^{k}\right| \cdot\left(\sup _{i . k}\left|\tilde{m}_{i}^{k}\right|\right)^{-1}
\end{aligned}
$$

where the indexes $i$ and $k$ cover $1, . ., L / h$ and $1, \ldots, Z / \Delta z$. Table 2 gives the error for different choices of the five coefficients $\sigma(i), i=1, . ., 5$ and two locations of the source. In any case the error is roughly several tenth of a percent while the error for the Dirichlet condition is from 6 to 29 per cent. These errors have to be compared with those due to the dispersion of the scheme. The reference solution has been recomputed with all the discretization steps divided by a factor 6 thus allowing us to estimate the relative error of the dispersion effects. We have found a relative error of $1.1 \%$ for $\epsilon_{\infty}$ and 0.4 $\%$ for $\epsilon_{2}$ which is of the same magnitude than the error due to the PMLs.

RR no2964 


\begin{tabular}{c||c|c|c|c|c|c|}
\cline { 2 - 7 } & Dirichlet & $N=7$ & $N=10$ & $N=15$ & $N=20$ & $N=40$ \\
\hline$\epsilon_{2}$ & $6.4 \%$ & $0.21 \%$ & $0.17 \%$ & $0.16 \%$ & $0.16 \%$ & $0.17 \%$ \\
\hline$\epsilon_{\infty}$ & $4.8 \%$ & $0.15 \%$ & $0.11 \%$ & $0.08 \%$ & $0.08 \%$ & $0.10 \%$ \\
\hline
\end{tabular}

$\underline{\text { Source } x_{s}=50 h}$

\begin{tabular}{c||c|c|c|c|c|c|}
\cline { 2 - 7 } \multicolumn{1}{c||}{} & Dirichlet & $N=7$ & $N=10$ & $N=15$ & $N=20$ & $N=40$ \\
\hline$\epsilon_{2}$ & $22 \%$ & $0.71 \%$ & $0.64 \%$ & $0.64 \%$ & $0.62 \%$ & $0.54 \%$ \\
\hline$\epsilon_{\infty}$ & $29 \%$ & $1.04 \%$ & $1.00 \%$ & $1.11 \%$ & $1.12 \%$ & $1.12 \%$ \\
\hline
\end{tabular}

Source $x_{s}=15 h$

Table 2: $L^{2}$ norm $\left(\epsilon_{2}\right)$ and $L^{\infty}$ norm $\left(\epsilon_{\infty}\right)$ of the relative error due to the lateral boundary condition. Comparison between the Dirichlet boundary condition and the PML with the coefficients optimized for a specific value of $N$, the number of points per wavelength.

We address now the question of the ability of the PML to absorb the waves propagating close to the vertical. For this, new experiments with a domain of interest of 32 points in the $x$ direction and 200 points in the $z$ direction are considered. In such a domain, waves propagate quasi-vertically and dispersion effects are attenuated allowing us to use half as many points per wavelength as in the previous experiments (the frequency of the signal, $2 \pi \omega_{s}$, is chosen equal to $20 / t_{s}$ ).

In Figure 6, we compare the solution computed in a large domain (320h) with solutions computed in smaller domains, namely $42 h$ or $52 h$. On both boundaries a Dirichlet condition or a PML condition with 5 or 10 points in the layer is imposed. The coefficients of the layer are the optimized coefficients for $N=5$ (see table 3 ). We can see that the spurious reflexions due to the Dirichlet boundary condition are greatly attenuated. This is confirmed by the evaluation of the errors in Table (5). The error drops from $98 \%$ (Dirichlet) to $3 \%$ (5h PML) or $0.4 \%$ (10h PML).

Finally, we test the ability of the method to deal with heterogeneous media. The test, graciously realized by E.Bécache, ([3]), is the migration of a point 


\begin{tabular}{|c||c|c|}
\hline \multicolumn{1}{|c||}{$N=5$} & \multicolumn{2}{c|}{$N=10$} \\
\hline$\sigma \Delta x(1)=0.185$ & $\sigma \Delta x(1)=0.0186$ & $\sigma \Delta x(6)=1.23108$ \\
\hline$\sigma \Delta x(2)=0.652$ & $\sigma \Delta x(2)=0.08473$ & $\sigma \Delta x(7)=1.8979$ \\
\hline$\sigma \Delta x(3)=1.539$ & $\sigma \Delta x(3)=0.22194$ & $\sigma \Delta x(8)=2.96433$ \\
\hline$\sigma \Delta x(4)=3.424$ & $\sigma \Delta x(4)=0.44532$ & $\sigma \Delta x(9)=4.73401$ \\
\hline$\sigma \Delta x(5)=9.909$ & $\sigma \Delta x(5)=0.77180$ & $\sigma \Delta x(10)=10.0447$ \\
\hline
\end{tabular}

Table 3: Values of the optimized coefficients associated to $N=5$ for $5 h$ and for $10 h$ PML.

\begin{tabular}{c||c|c|c|}
\cline { 2 - 4 } \multicolumn{1}{c||}{} & Dirichlet & $\sigma_{5}$ & $\sigma_{10}$ \\
\hline$\epsilon_{2}$ & $82 \%$ & $2.3 \%$ & $0.41 \%$ \\
\hline$\epsilon_{\infty}$ & $92 \%$ & $2.6 \%$ & $0.37 \%$ \\
\hline
\end{tabular}

Table 4: $L^{2}$ norm $\left(\epsilon_{2}\right)$ and $L^{\infty}$ norm $\left(\epsilon_{\infty}\right)$ of the relative error due to the lateral boundary condition. Comparison between the Dirichlet boundary condition and the PML with the coefficients optimized for $N=5$. The length of the right and left layers is $5 h$ and $10 h$.

RR no2964 
F. Collino

source in a media with velocity law described in figure 7 . The improvement between the Dirichlet condition (figure 8, left) and the PML technic (figure 8 right), left) demonstrates that the method can also handle heterogeneous media very efficiently.

\section{Conclusion}

We have designed and analyzed a new absorbing layer model for the wide angle paraxial equations. The method is based on an interpretation and an extension of the Bérenger perfectly matched layers for Maxwell's equations. The model is parameterized by a damping function whose optimal choice is linked to the discretization step. A discrete plane wave analysis, which determines the reflection coefficient, shows the important role played by the discretization. We obtain an optimal damping function by minimizing this coefficient for all the angles of propagation. The efficiency of the method has been demonstrated by numerical experiments. Finally, let us note that the same method can be used for the artificial condition in the Schrodinger equation. This is precisely the subject of [15] that deals with the beam propagation method for applications in optics.

\section{APPENDIX}

\section{Appendix A: derivation of the elementary solution}

According to [7], the elementary solution of

$$
i \partial_{t} E+\Delta E=-\delta(x) \delta(t)
$$

is

$$
E(x, y)=\sqrt{\frac{i}{4 \pi t}} e^{i \frac{x^{2}}{4 z}} 1_{\{t>0\}}
$$

We look for the solution of

$$
2 i \omega \partial_{z} v-\Delta v=\delta(x) \delta(z)
$$


$w=\bar{v}$ satisfies

$$
2 i \omega \partial_{z} w+\Delta w=-\delta(x) \delta(z)
$$

or

$$
i \partial_{z / 2 \omega} w+\Delta w=-\delta(x) \delta\left(\frac{z}{2 \omega}\right) \frac{1}{2 \omega}
$$

Then

$$
v(x, z)=\bar{w}(x, z)=\frac{1}{2 \omega} \bar{E}(x, z)=\sqrt{\frac{-i}{8 \pi \omega z}} e^{-i \omega \frac{x^{2}}{2 z}} 1_{\{z>0\}}
$$

\section{Appendix B: Bérenger's PML and change of variable}

In [4], Bérenger proposes a new layer model, called a perfectly matched layer (PML) for electromagnetic waves. In this appendix, we show how Bérenger's PML can be viewed as a complex change of variable applied to Maxwell's system.

For the 2D Maxwell equations, the design of the PML in the region $x>0$ is as follows. The propagation of a transverse electric wave is governed by $\left(\epsilon_{0}=\mu_{0}=1\right)$

$$
\left\{\begin{array}{l}
\frac{\partial H_{z}}{\partial t}=\frac{\partial E_{x}}{\partial y}-\frac{\partial E_{y}}{\partial x} \\
\frac{\partial E_{y}}{\partial t}=-\frac{\partial H_{z}}{\partial x}, \quad \frac{\partial E_{x}}{\partial t}=\frac{\partial H_{z}}{\partial y} .
\end{array}\right.
$$

The construction of an absorbing layer in the right half space (i.e. in the region $x>0)$ is performed in two steps. First, we split the magnetic component into two parts as follows

$$
H_{z}=H_{z x}+H_{z y}
$$

and rewrite equations $(88)$ as

$$
\begin{cases}\frac{\partial H_{z y}}{\partial t}=\frac{\partial E_{x}}{\partial y}, & \frac{\partial H_{z x}}{\partial t}=-\frac{\partial E_{y}}{\partial x} \\ \frac{\partial E_{y}}{\partial t}=-\frac{\partial H_{z}}{\partial x}, & \frac{\partial E_{x}}{\partial t}=\frac{\partial H_{z}}{\partial y}\end{cases}
$$

At this stage, equations (89) and (90) are equivalent to the initial problem.

RR no2964 
In a second step, a damping factor $\sigma(x),(\sigma(x)=0$ for $x<0)$ is introduced in each equation wherever the normal derivative operator $\partial_{x}$ appears :

$$
\left\{\begin{array}{l}
\frac{\partial H_{z y}}{\partial t}=\frac{\partial E_{x}}{\partial y}, \quad \frac{\partial H_{z x}}{\partial t}+\sigma H_{z x}=-\frac{\partial E_{y}}{\partial x} \\
\frac{\partial E_{y}}{\partial t}+\sigma E_{y}=-\frac{\partial H_{z}}{\partial x}, \quad \frac{\partial E_{x}}{\partial t}=\frac{\partial H_{z}}{\partial y}
\end{array}\right.
$$

System (91) is Bérenger's PML model.

If we look for the stationary solutions with frequency $\omega$, we get

$$
\left\{\begin{array}{l}
i \omega \hat{H}_{z y}=\frac{\partial \hat{E}_{x}}{\partial y}, \quad(i \omega+\sigma(x)) \hat{H}_{z x}=-\frac{\partial \hat{E}_{y}}{\partial x} \\
(i \omega+\sigma(x)) \hat{E}_{y}=-\frac{\partial \hat{H}_{z}}{\partial x}, \quad i \omega \hat{E}_{x}=\frac{\partial \hat{H}_{z}}{\partial y}, \quad \hat{H}_{z}=\hat{H}_{z x}+\hat{H}_{z y}
\end{array}\right.
$$

We can rewrite this system of equations with the only unknown $\hat{H}_{z}$. We have

$$
(i \omega)^{2}\left(\hat{H}_{z x}+\hat{H}_{z y}\right)-i \omega \frac{\partial \hat{E}_{x}}{\partial y}+\frac{(i \omega)^{2}}{i \omega+\sigma(x)} \frac{\partial \hat{E}_{y}}{\partial x}=0
$$

or

$$
\omega^{2} \hat{H}_{z}+\frac{\partial^{2} \hat{H}_{z}}{\partial y^{2}}+\frac{i \omega}{i \omega+\sigma(x)} \frac{\partial}{\partial x}\left(\frac{i \omega}{i \omega+\sigma(x)} \frac{\partial \hat{H}_{z}}{\partial x}\right)=0 .
$$

In the region where $\sigma$ is zero, we recover the well known property that, the magnetic field of a non-stationary 2D transverse electric wave satisfies the Helmoltz equation. Inside the layer, we remark that the PML model consists in the simple substitution

$$
\frac{\partial}{\partial x} \longrightarrow \frac{\partial}{\partial \tilde{x}}=\frac{i \omega}{i \omega+\sigma(x)} \frac{\partial}{\partial x}
$$

where

$$
\tilde{x}=x-\frac{i}{\omega} \int_{0}^{x} \sigma(\xi) d \xi
$$

in the initial equation, whereas the tangential derivatives are left unchanged. A direct inspection shows that the Green function for equation (93) is

$$
\hat{H}_{z}(x, z)=\frac{i}{4} H_{0}^{(1)}\left(\omega \sqrt{\tilde{x}^{2}+y^{2}}\right) \quad\left(\Rightarrow\left|H_{z}(x, z)\right| \sim \frac{C}{\sqrt{\omega \tilde{x}}} e^{-\int_{0}^{x} \sigma(\xi) d \xi} x \rightarrow+\infty\right)
$$


where $H_{0}^{(1)}$ is the Hankel function of the first kind.

This is one key to understanding the nice properties of the PML model. This model is simply obtained using the complex change in variables (95) applied to the original equation. The right line $x \in R$ is moved into a path in the upper complex plane. The existence of an analytical extension of the Green's function in the upper complex plane allows us to properly define $\hat{H}_{z}$ and the exponential decay of this extension ensures the damping effect.

\section{Appendix C: derivation of the interface conditions}

Let us suppose that $\sigma(x)$ is a positive function, vanishing for $x<0$ and continuous for $x>0$. At $x=0, \sigma(x)$ is supposed to have a right limit not necessarily equal to 0 ,

$$
\lim _{x \rightarrow 0^{+}} \sigma(x)=\sigma\left(0^{+}\right) \geq 0,
$$

In section 1 , we were interested in determining of particular solutions to

$$
2 i \omega \partial_{z} v+\frac{i \omega}{i \omega+\sigma} \partial_{x}\left(\frac{i \omega}{i \omega+\sigma} \partial_{x} v\right)=0
$$

A first problem is to make sense of this equation as $\sigma$ is not continuous at $x=0$. We first rewrite equation (96) as

$$
\frac{2 i \omega}{d(x)} \partial_{z} v(x, z)+\partial_{x}\left(d(x) \partial_{x} v\right)(x, z)=0, \quad d(x)=\frac{i \omega}{i \omega+\sigma(x)},
$$

and we call solution of (97) a function $v$ such that

1. $v(x, z)$ is continuous in $x$ and $z$,

2. The $x$ derivative of $v(x, z)$ exists and is a continuous function in $x \neq 0$ with right and left limits at $x=0$.

3. $v(x, z)$ satisfies $(97)$ in the sense of distributions.

With this definition, the function $\varphi(x, z)=d(x) \partial_{x} v(x, z)$ is a continuous function except possibly at $x=0$ where it possesses right and left limits, so the second $x$-derivative in (97) make sense as a distribution derivative. For such a function, we have

RR no2964 


$$
\partial_{x} \varphi=\left(\partial_{x} \varphi\right)(x, z) \mathbf{1}_{\Omega^{+}}(x, z)+\left(\partial_{x} \varphi\right)(x, z) \mathbf{1}_{\Omega^{-}}(x, z)+\left(\varphi\left(0^{+}\right)-\varphi\left(0^{-}\right)\right) \delta(x),
$$

where $\mathbf{1}_{\Omega^{+}}$, is 1 on $\Omega^{+}=\{(x, z), x>0\}$ and 0 on $\Omega^{-}=\{(x, z), x<0\}, \mathbf{1}_{\Omega^{-}}=$ $1-\mathbf{1}_{\Omega^{+}}$and $\delta(x)$ is the Dirac distribution.

Now, the function

$$
\begin{cases}\tilde{v}(x, z)=\left(e^{-i k_{x} x}+R e^{i k_{x} x}\right) e^{i k_{z} z} & x<0 \\ \tilde{v}(x, z)=T e^{-i k_{x} \int_{0}^{x} d^{-1}(\xi) d \xi} e^{i k_{z} z} & x>0 \\ \text { with } \quad k_{x}^{2}=-2 \omega k_{z}, \quad k_{x}>0, & \end{cases}
$$

has the required regularity when $T=1+R$ (continuity). It satisfies

$$
\frac{2 i \omega}{d(x)} \partial_{z} \tilde{v}+\partial_{x}\left(d(x) \partial_{x} \tilde{v}\right)=\left(d\left(0^{+}\right) \partial_{x} \tilde{v}\left(0^{+}, z\right)-d\left(0^{-}\right) \partial_{x} \tilde{v}\left(0^{-}, z\right)\right) \delta(x) .
$$

Thus, $\tilde{v}$ satisfies equation (97) in the distributional sense if and only if

$$
d\left(0^{+}\right) \partial_{x} \tilde{v}\left(0^{+}, z\right)=d\left(0^{-}\right) \partial_{x} v\left(0^{-}, z\right) .
$$

This condition is the second transmission condition.

\section{Appendix D: Estimations for the case $d(x)=C^{s t e}$}

We consider the approximation of problem (30), (31) obtained by taking a constant value for $\sigma_{0}$, namely

$$
\left\{\begin{array}{l}
\text { Find } \left.u_{h}: z \in\right] 0, Z\left[\longrightarrow u(., z) \in V_{h} \quad \text { such that } \forall v_{h}(x) \in V_{h},\right. \\
2 i \omega \int \partial_{z} u_{h}(x, z) v_{h}(x) \frac{d x}{d_{0}}=-\int \partial_{x} u_{h}(x, z) \partial_{x} v_{h}(x) d_{0} d x \\
u_{h}(z=0)=u_{h}^{0} \in V_{h}, \quad \text { with } d_{0}=\frac{i \omega}{i \omega+\sigma_{0}}, \quad \sigma_{0} \geq 0 .
\end{array}\right.
$$


Substituting for $v_{h}$ successively by the conjugate of $u_{h}$ and $\partial_{z} u_{h}$, we get

$$
\begin{aligned}
& \left\{\begin{aligned}
\frac{d}{d z}\left(\int\left|u_{h}\right|^{2} d x\right) & =\frac{1}{2} \Re\left(\int \partial_{z} u_{h} \overline{u_{h}} d x\right) \\
& =-\Re\left(\frac{d_{0}^{2}}{4 i \omega}\right) \int\left|\partial_{x} u_{h}\right|^{2} d x,
\end{aligned}\right. \\
& \left\{\begin{aligned}
\frac{d}{d z}\left(\int\left|\partial_{x} u_{h}\right|^{2} d x\right) & =\frac{1}{2} \Re\left(\int \partial_{x} u_{h} \partial_{x} \overline{\partial_{z} u_{h}} d x\right) \\
& =-\Re\left(\frac{i \omega}{d_{0}^{2}}\right) \int\left|\partial_{z} u_{h}\right|^{2} d x,
\end{aligned}\right.
\end{aligned}
$$

where $\Re$ stands for the real part, Now, we have

$$
\Re\left(\frac{i \omega}{d_{0}^{2}}\right)=2 \sigma_{0} \geq 0, \quad \Re\left(\frac{d_{0}^{2}}{4 i \omega}\right)=1 /\left(8 \sigma_{0}\right) \geq 0,
$$

which implies

$$
\begin{aligned}
\frac{d}{d z}\left(\int\left|u_{h}\right|^{2} d x\right), \frac{d}{d z}\left(\int\left|\partial u_{h}\right|^{2} d x\right) \leq 0 \\
\quad\left(\int\left|u_{h}\right|^{2} d x\right)(z) \leq\left(\int\left|u_{h}^{0}\right|^{2} d x\right) \\
\Rightarrow \quad\left(\int\left|\partial_{x} u_{h}\right|^{2} d x\right)(z) \leq\left(\int\left|\partial_{x} u_{h}^{0}\right|^{2} d x .\right)
\end{aligned}
$$

These estimations allow us to use the standard Galerkin method and to prove that $u_{h}$ has a limit, as $h$ goes to zero, and is continuous in $z$ with values in $V$ (cf [7]). Of course, the same estimations hold for every solution of problem (30) which establishes uniqueness. Let us remark that this result is very poor, as we used the fact that $\sigma$ was constant in the whole domain. Its is of interest in that it points out the importance of the positiveness of $\sigma$ for the well-posedness of the problem.

RR no2964 


\section{Appendix E: assembly of the matrices for the discrete problem}

This appendix is devoted to the evaluation of

$$
\mathbf{M}_{i, j}=\int_{R} \hat{v}_{i}(x) \hat{v}_{j}(x) \frac{d x}{d(x)}, \quad \mathbf{K}_{i, j}=\int_{R} \partial_{x} \hat{v}_{i}(x) \partial_{x} \hat{v}_{j}(x) d(x) d x
$$

where $d(x)$ is assumed constant on each interval $\left.I_{i+\frac{1}{2}}=\right] i \Delta x,(i+1) \Delta x[=$ ]$x_{i}, x_{i+1}\left[\right.$, and $\hat{v}_{i}$ is the hat function

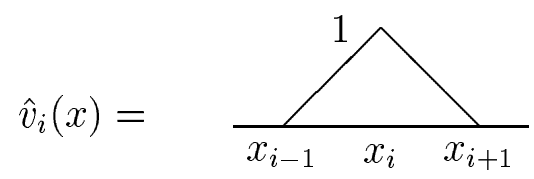

We start from

$$
\int_{R} \hat{v}_{i}(x) \hat{v}_{j}(x) \frac{d x}{d(x)}=\sum_{m} \frac{1}{d_{m+\frac{1}{2}}} \int_{I_{m+\frac{1}{2}}} \hat{v}_{i}(x) \hat{v}_{j}(x) d x .
$$

An exact computation gives

$$
\left\{\begin{array}{lll}
\int_{I_{m+\frac{1}{2}}} \hat{v}_{i}(x) \hat{v}_{i}(x) d x & =\frac{\Delta x}{3} \text { if } m=i, i-1 \\
\int_{I_{m \pm \frac{1}{2}}} \hat{v}_{i}(x) \hat{v}_{i \pm 1}(x) d x=\frac{\Delta x}{6} & \text { if } m=i \\
\int_{I_{m+\frac{1}{2}}} \hat{v}_{i}(x) \hat{v}_{j}(x) d x & =0 & \text { elsewhere }
\end{array}\right.
$$

while a two-points quadrature rule provides

$$
\left\{\begin{array}{lll}
\int_{I_{m+\frac{1}{2}}} \hat{v}_{i}(x) \hat{v}_{i}(x) d x & \approx \frac{\Delta x}{2} \text { if } m=i, i-1 \\
\int_{I_{m+\frac{1}{2}}} \hat{v}_{i}(x) \hat{v}_{j}(x) d x & \approx 0 & \text { elsewhere. }
\end{array}\right.
$$


Taking a linear combination of (105) and (106) with respective weight $6 \gamma$ and $1-6 \gamma$ gives

$$
\left\{\begin{array}{l}
\int_{R} \hat{v}_{i}(x) \hat{v}_{i}(x) d x \approx(1-2 \gamma) \frac{\Delta x}{2}\left(\frac{1}{d_{i+\frac{1}{2}}}+\frac{1}{d_{i-\frac{1}{2}}}\right) \\
\int_{R} \hat{v}_{i}(x) \hat{v}_{i \pm 1}(x) d x \approx \gamma \frac{\Delta x}{d_{i \pm \frac{1}{2}}} \\
\int_{R} \hat{v}_{i}(x) \hat{v}_{j}(x) d x=0 \text { if }|i-j|>1 .
\end{array}\right.
$$

In the same way

$$
\int_{R} \partial_{x} \hat{v}_{i}(x) \partial_{x} \hat{v}_{j}(x) d(x) d x=\sum_{m} d_{m+\frac{1}{2}} \int_{I_{m+\frac{1}{2}}} \partial_{x} \hat{v}_{i}(x) \partial_{x} \hat{v}_{j}(x) d x,
$$

with

$$
\left\{\begin{array}{lll}
\int_{I_{m+\frac{1}{2}}} \partial_{x} \hat{v}_{i}(x) \partial_{x} \hat{v}_{i}(x) d x & =\frac{1}{\Delta x} & \text { if } m=i, i-1 \\
\int_{I_{m \pm \frac{1}{2}}} \partial_{x} \hat{v}_{i}(x) \partial_{x} \hat{v}_{i \pm 1}(x) d x & =-\frac{1}{\Delta x} & \text { if } m=i \\
\int_{I_{m+\frac{1}{2}}} \partial_{x} \hat{v}_{i}(x) \partial_{x} \hat{v}_{j}(x) d x & =0 & \text { elsewhere }
\end{array}\right.
$$

and so

$$
\left\{\begin{array}{l}
\int_{R} d(x) \partial_{x} \hat{v}_{i}(x) \partial_{x} \hat{v}_{i}(x) d x=\frac{1}{\Delta x}\left(d_{i+\frac{1}{2}}+d_{i-\frac{1}{2}}\right) \\
\int_{R} d(x) \partial_{x} \hat{v}_{i}(x) \partial_{x} \hat{v}_{i \pm 1}(x) d x=-\frac{d_{i \pm \frac{1}{2}}}{\Delta x} \\
\int_{R} d(x) \partial_{x} \hat{v}_{i}(x) \partial_{x} \hat{v}_{j}(x) d x=0 \text { if }|i-j|>1
\end{array}\right.
$$

\section{References}

[1] L. Halpern A. Bamberger, B. Engquist and P. Joly. Higher order paraxial approximations for the wave equation. Siam J. Appl. Math., pages 128154, 1988.

RR no2964 
F. Collino

[2] V.A. Basbakov and A.V. Popov. Implementation of transparent boundaries for numerical solution of the Schrödinger Equation. Wave Motion., 14:123-128, 1991.

[3] E. Bécache, F. Collino, and Patrick Joly. Higher order numerical schemes for paraxial approximations of the wave equation. In 2nd International Conference on Theoretical Computational Acoustics, 1995.

[4] J.P. Bérenger. A perfectly matched layer for the absorption of electromanetic waves. Journal of Comp. Physics., 114:185-200, 1994.

[5] J. F. Claerbout. Imaging the earth's interior. Blackwell Scientific Publication co., 1983.

[6] F. Collino. Numerical method for 2-d migration. Annual Report, PSI Consortium, IFP, Rueil-Malmaison, France, 1993.

[7] R. Dautrey and J.L.Lions. Analyse mathématique et calcul numérique pour les sciences et les techniques, volume 7. Masson, 1988.

[8] B. Dimenza. Approximations numériques d'équations de Schrodinger non liméaires et de modèles associés. PhD thesis, Université de Bordeaux 1, June 1995.

[9] A. A. Dubrulle. On numerical method for migration in layered media. Geophysical Prospecting, 56(11):237-264, 1983.

[10] D.M. Eidus. The principle of limiting absorption. Amer. Math. Soc. Transl., 47:157-191, 1965.

[11] C. Lemaréchal. module m2qn1: document modulopt. Technical report, I.N.R.I.A., Domaine de de Voluceau Rocquencourt, B.P.105, 78153, Le Chesnay Cedex France, 1983.

[12] S. Marcus. A Generalized impedance method for application of the parabolic approximation to underwater acoustics . J.Acoust. Soc. Am.., 90(1):391-398, 1991. 
[13] M.Israeli and S.A. Orszag. Approximation of radiation boundary conditions. Journal of Comp. Physics., 41:115-135, 1981.

[14] C.M. Rappaport. Perfectly Matched Absorbing Conditions Based on Anisotropic Lossy Mapping of Space. IEEE Microwave and Guided Wave Letters., 5(3):90-92, 1995.

[15] C. Vasallo and F. Collino. Higly efficient absorbing boundary conditions for the beam propagation method. IEEE J. of Lightwave Technology, 14(6):1570-1577, June 1996.

RR no2964 


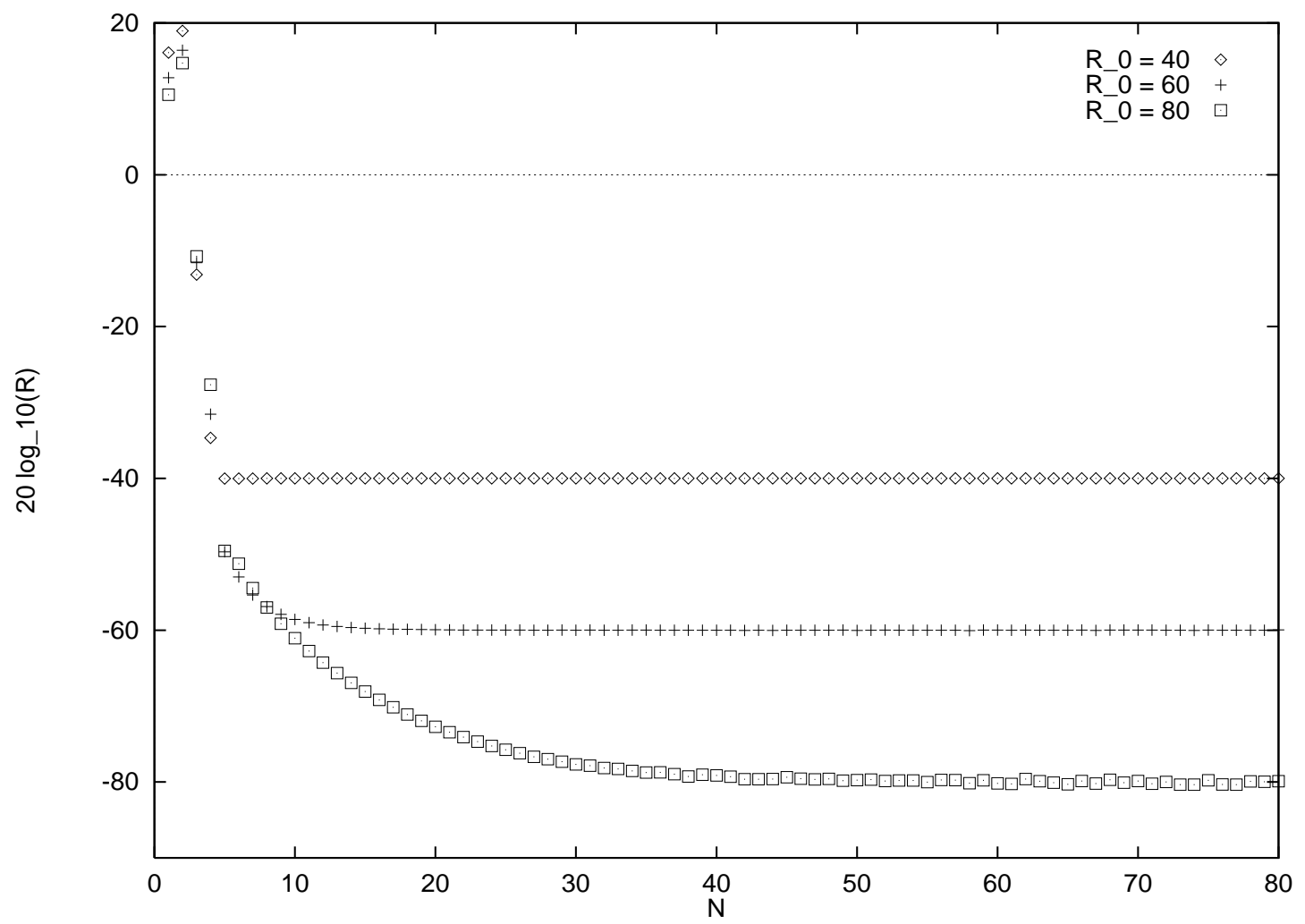

Figure 1: Numerical reflection coefficient (in Db, i.e. $20 \log _{10} R$ ) versus the number of points per wavelength for three different values of $R_{0}, R_{0}=10^{-2}$ $(-40 \mathrm{db}), 10^{-3}$ (-60db), $10^{-4}(-80 \mathrm{db})$. 


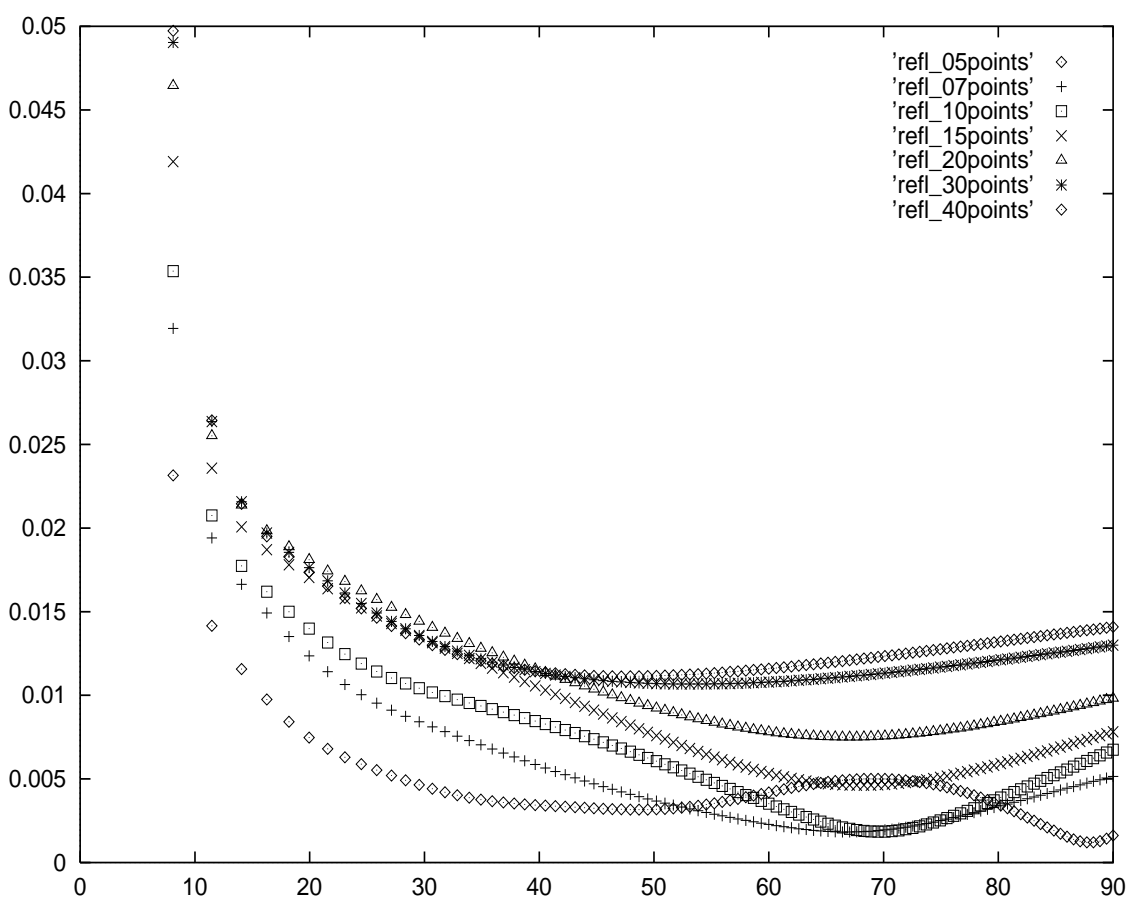

Figure 2: Reflection coefficient versus the angle $\theta,\left(k_{z}^{\text {true }}=\omega \cos \theta\right)$ for different choices of the number of points per wavelength. Each curve corresponds to the 5 coefficients of the PML given in Table 1

RR no2964 


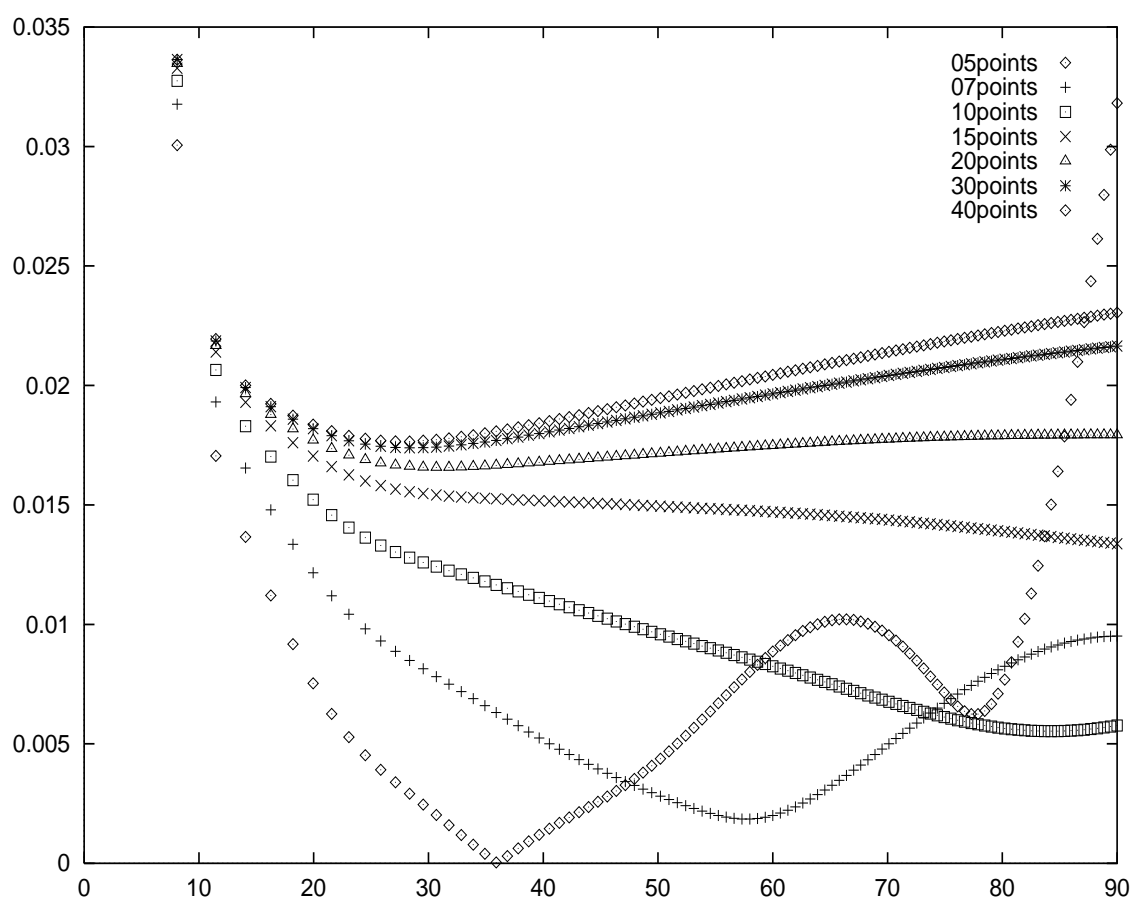

Figure 3: Reflexion coefficient versus the angle $\theta$, for different choices of the number of points per wavelength. The 5 coefficients of the PML are given in Table 1 in the column $N=7$ 

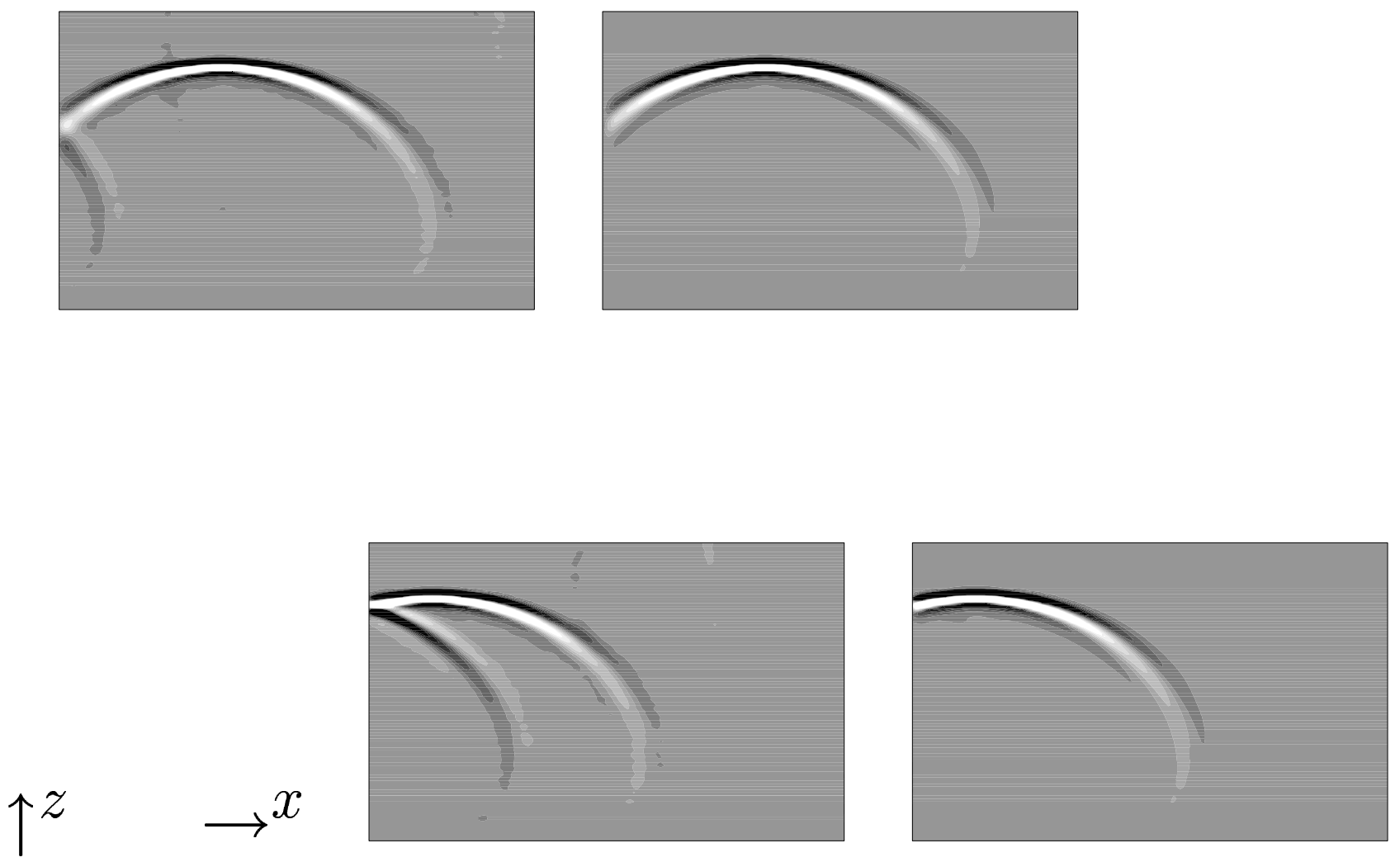

Figure 4: Migration of a point source with the 45 degree paraxial equation. The source is located at $50 \Delta x$ (top) or $15 \Delta x$ (bottom). At the left boundary is a Dirichlet boundary condition (left) or an absorbing layer composed with 4 extra nodes (right). 

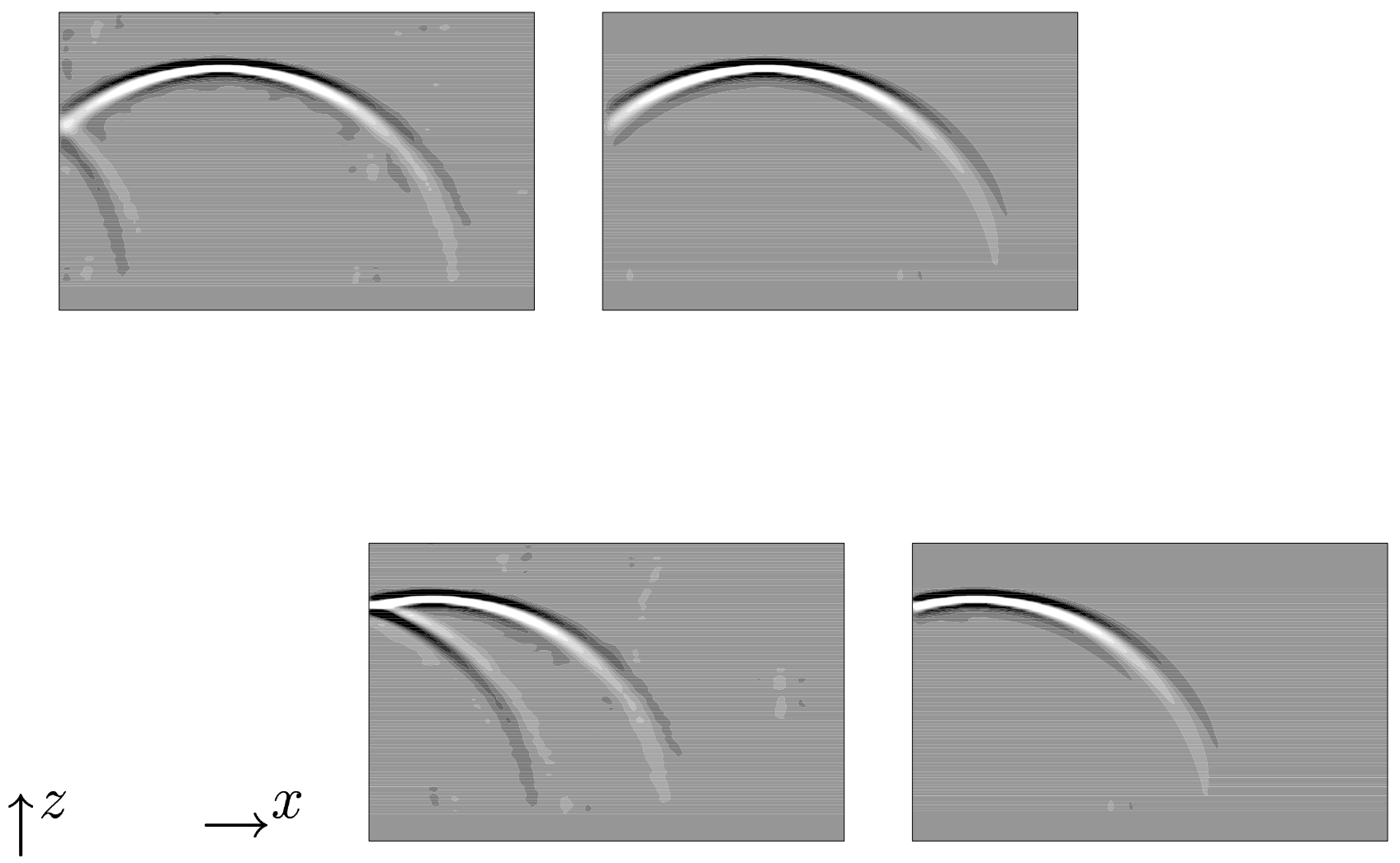

Figure 5: Migration of a point source with the 60 degree paraxial equation. The source is located at $50 \Delta x$ (top) or $15 \Delta x$ (bottom). At the left boundary is a Dirichlet boundary condition (left) or an absorbing layer composed with 4 extra nodes (right). 

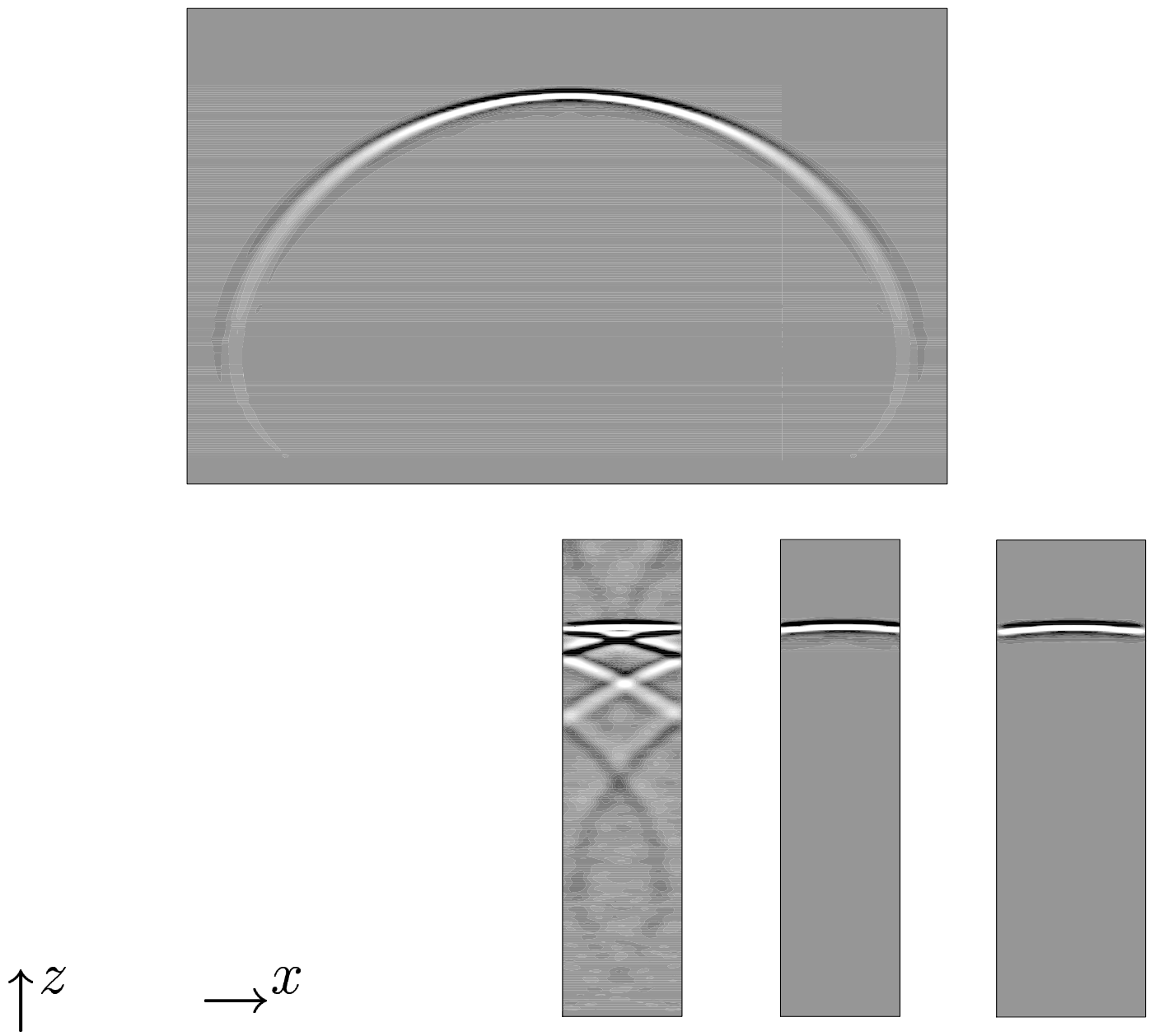

Figure 6: Comparison between the reference solution computed in the domain $320 h \times 200 \Delta z$ (top) and three solutions computed in a $42 h \times 200 \Delta z$ (bottom left and bottom center) or a $52 h \times 200 \Delta z$ (bottom right) domain. The source is located in the center of each domain. Bottom left corresponds to a Dirichlet boundary condition, bottom center to a $5 h$ PML and bottom right to a $10 h$ PML. 


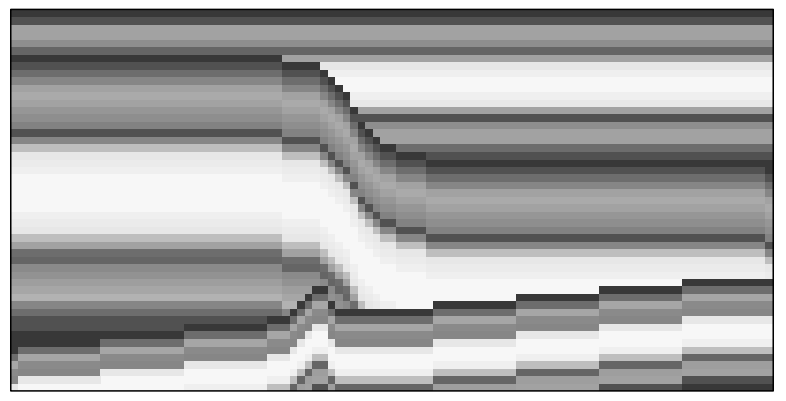

Figure 7: 2D smooth heterogeneous medium
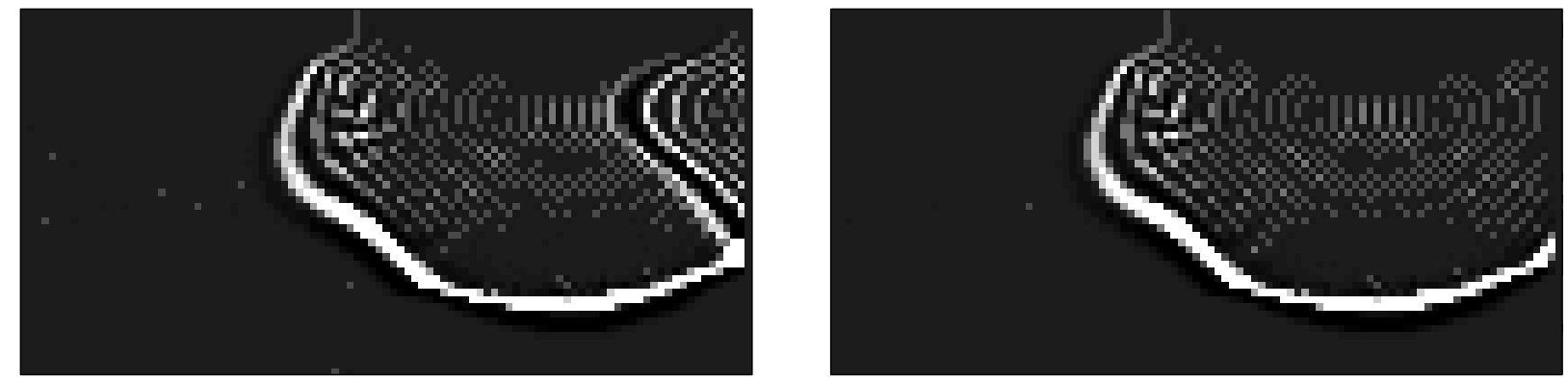

Figure 8: Migration of a filtered point source in a 2D smooth heterogeneous medium. 


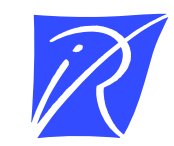

Unité de recherche INRIA Lorraine, Technopôle de Nancy-Brabois, Campus scientifique, 615 rue du Jardin Botanique, BP 101, 54600 VILLERS LÈS NANCY

Unité de recherche INRIA Rennes, Irisa, Campus universitaire de Beaulieu, 35042 RENNES Cedex

Unité de recherche INRIA Rhône-Alpes, 655, avenue de l'Europe, 38330 MONTBONNOT ST MARTIN

Unité de recherche INRIA Rocquencourt, Domaine de Voluceau, Rocquencourt, BP 105, 78153 LE CHESNAY Cedex

Unité de recherche INRIA Sophia-Antipolis, 2004 route des Lucioles, BP 93, 06902 SOPHIA-ANTIPOLIS Cedex

Éditeur

INRIA, Domaine de Voluceau, Rocquencourt, BP 105, 78153 LE CHESNAY Cedex (France)

ISSN 0249-6399 